\title{
ESTRUCTURAS DE COMBUSTIÓN EN EL ENTORNO DE LA SIERRA DE FONTCALENT (ALACANT): UN PRIMER ACERCAMIENTO A SU ESTUDIO ${ }^{1}$
}

\author{
FURNACES IN THE SIERRA OF FONTCALENT (ALICANTE): A PRELIMINARY STUDY
}

\author{
JORDI A. LÓPEZ LILLO \\ Universidad de Alicante
}

\section{INTRODUCCIÓN}

El objeto del presente trabajo es una serie de estructuras relacionadas, sin duda, con el uso del fuego para la transformación de un elemento en principio desconocido; por tanto, y de forma genérica, de una serie de hornos. Se localizan en una amplia zona de la parte noroccidental de la comarca de l'Alacantí, delimitada por las sierras de Fontcalent, Mitjana y les Àguiles por el norte, hasta el trazado de la actual A-31 por el sur (Fig. 1), y sin aparente vinculación con otros restos de origen antrópico, es decir, alejada de las áreas de población actuales o los sitios arqueológicos y con una dudosa relación con los materiales muebles que, eventualmente, pueden encontrarse en sus proximidades. Pero quizá la particularidad más determinante a la hora de abordar su estudio sea la ausencia de registro previo. Así, no sólo no están catalogadas en el inventario de yacimientos arqueológicos de la Direcció General de Patrimoni Cultural Valencià, sino que tampoco se las ha documentado en los trabajos arqueológicos previos realizados en esta área ${ }^{2}$, lo que se viene a sumar al silencio de las fuentes históricas tradicionales. De hecho, ni siquiera han sido identificadas y reconocidas

1. Este estudio corresponde, salvo pequeñas adaptaciones para su publicación, al Trabajo de Investigación Tutelado por el Prof. Jesús Moratalla Jávega para el Màster Oficial en Arqueologia Professional de la Universitat d'Alacant durante el curso 2008-2009. Aparte de al equipo docente, he de agradecer especialmente la ayuda de Javier Franco, Patrice Cressier y Jacques Thiriot, tanto como la de los compañeros que colaboraron en el trabajo de campo, Ximo Martorell, Adela Sánchez y Lourdes Rebollo, sin que la responsabilidad de los errores que aparezcan a continuación sea más que mía.

2. Por otra parte, más allá de las intervenciones en los sitios arqueológicos previamente catalogados, éstos se reducen a algunas prospecciones como la EDAR Alacant Sur, al este de Fontcalent, y dirigida por M. A. Esquembre y J. Boronat, la realizada por D. Serrano y A. Valero Climent en la finca Lo Boligni, o las vinculadas con las obras del AVE (cfr. AA.VV. 2008a y b). por los trabajadores agrícolas de las contornadas con quienes hemos podido hablar a lo largo del trabajo de campo. Todo ello, por supuesto, sin más implicaciones que la obvia: que nuestro punto de partida será el desconocimiento.

Siendo de esta forma, vamos a descubrir su existencia a través de la información proporcionada por operarios que trabajaban en el mantenimiento de los tendidos eléctricos, lo que les obligaba a frecuentar la zona, las más de las veces fuera de los ejes principales de comunicación; será en estas visitas en las que se aperciban de unas estructuras diseminadas por el campo, en agrupaciones a veces muy distantes entre sí, y que «aparecen y desaparecen con las lluvias». De esta descripción se extrae sin demasiados problemas, primero, un alto factor de riesgo para su conservación frente a la erosión usual a que están sometidos los restos, principalmente materializada en la fuerte afección de las puntuales pero intensas precipitaciones y las torrenteras que provocan, como veremos. En segundo lugar, se puede presuponer la existencia de un buen número de estas estructuras en origen, como mínimo el suficiente para que la pérdida continua de los restos superficiales se haya compensado con la puesta al descubierto de otros, de manera que se ha mantenido un cómputo tal de evidencias de su presencia que, en el momento de iniciar este trabajo, todavía se han podido documentar más de quince hornos sólo visitando los puntos en que se había localizado concentraciones varios años atrás, de los cuales no en todos ha tenido éxito la prospección.

Opinamos que todas estas circunstancias redundan en la necesidad de abordar el estudio de unos elementos recurrentes pero desconocidos, cuya integridad y conservación, además, peligra gravemente. Al otro lado, la dificultad intrínseca que supone iniciar cualquier investigación va a verse acrecentada, en nuestro caso, por las especiales condiciones que plantea un registro realmente parco y una cierta limitación, marcada por el carácter mismo de este estudio y por los recursos materiales disponibles para llevarlo a cabo. 


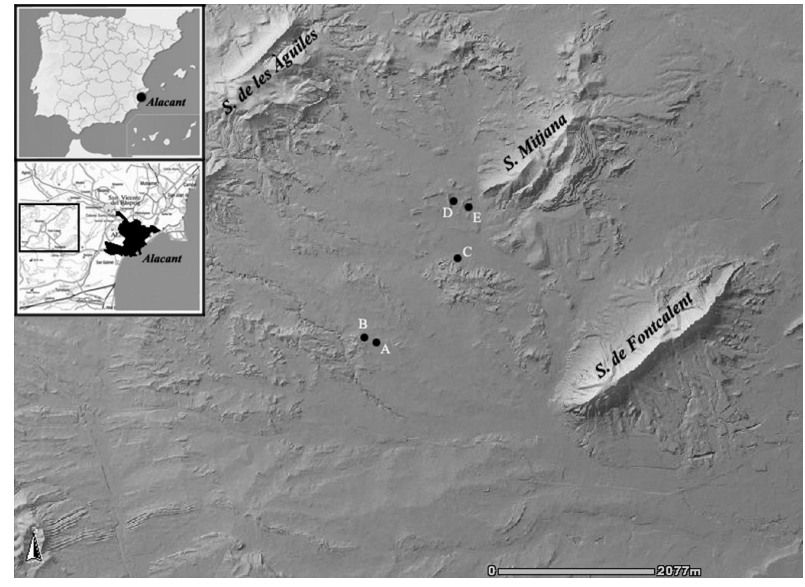

Figura 1: Mapa de situación de las zonas con agrupaciones de hornos.

Pero un enfoque acertado de estas condiciones, a la vez, va a permitirnos delimitar un objetivo claro y ajustado a ellas, que es la única manera de construir un conocimiento arqueológico efectivo y capaz de contribuir a ulteriores trabajos.

Así, en las siguientes páginas se trata de aportar un primer caudal de información para el conocimiento de las estructuras de combustión de Fontcalent, con lo que conlleva de carácter primera y fundamentalmente descriptivo. Acorde con esta intención, nos hemos limitado a documentar las evidencias arrojadas por una prospección asistémica, centrada, como decíamos, en los lugares donde los informantes recordaban haber visto estos hornos desde los caminos, intensificada y extendida al entorno inmediato de los puntos en que se ha verificado su presencia. La justificación de esta estrategia la da un número de localizaciones más que suficiente para nuestros fines, por más que no se haya podido aislar, en una segunda parte del estudio, una única explicación funcional que no sólo encaje con las pruebas, sino que permita, además, descartar satisfactoriamente otras caracterizaciones. Sin embargo, opinamos que esta circunstancia hace más interesante la exposición de unas hipótesis que únicamente podrán confirmarse o descartarse con una intervención arqueológica mayor, tal y como se evidencia en las conclusiones de este trabajo, habiendo puesto antes en relación nuestro registro con otros estudios sobre estructuras similares y ensayado el ajuste de los procesos productivos que pudieron haberlo generado al marco contextual, es decir, a las posibilidades históricas y económicas de la zona.

\section{LAS PARTIDAS RURALES NOROCCIDENTA- LES DEL CAMP D'ALACANT. CONTEXTO FÍSI- CO E HISTÓRICO}

Con sus 426 m s.n.m., y a tan sólo 11 km oeste-noroeste del núcleo urbano de Alacant, la mole de cerca de tres kilómetros longitudinales de la sierra de Fontcalent representa un hito paisajístico de primer orden, al ser la última elevación importante antes de llegar a la costa, de manera que es plenamente visible desde la fachada marítima y todo el glacis del Camp d'Alacant que desciende hasta ella. Este carácter, además, se va a ver reforzado por la abundancia de agua en sus inmediaciones, destacando el manantial termal que mana en su base y la laguna que hubo entre ella y la Mitjana, hoy reducida a saladar, y por su ubicación próxima a dos de los ejes de comunicación principales de la comarca, pues si, por un lado, el camino que conecta la ciudad con el interior montañoso a través de la Foia de Castalla mantiene la sierra inmediatamente al occidente en todo momento, por el otro, la comunicación con la Vall del Vinalopó -que equivale a decir con el interior peninsular- se ha realizado tradicionalmente pasando siempre cerca de la Fontcalent: así, encontramos allí desde el ramal que se desviaba a la costa desde la Vía Augusta hasta la actual autovía A-31 o la línea ferroviaria del AVE, todavía en ejecución. Pero, antes de llegar a la significación antrópica de la zona que nos ocupa, es necesario que acotemos su marco geológico, dadas las implicaciones que va a tener en nuestro estudio.

\section{CARACTERIZACIÓN GEOLÓGICA}

Tal y como recoge el Instituto Geológico y Minero (IGME, 1978), la sierra de Fontcalent, como la Mitjana, algo más septentrional, tiene su origen en los intensos movimientos de compresión que crearon una línea extrusiva jurásica, la cual, en esta parte, se rompe en una falla inversa, alterando la secuencia ordinaria para presentar casi en vertical una estratigrafía que cuenta con niveles más o menos impermeables del Trías por debajo de las dolomías, calizas y sílex jurásicos. De esta manera, se crea entre ambas elevaciones, y puesto que aquí va a verter todo el arco montañoso que cierra la comarca por el oeste, una depresión semiendorreica que desagua al occidente de Fontcalent por el Barranc de l'Infern hacia el de Aigua Amarga y el mar, y que permitió el sistema de marismas al norte de esta sierra que citábamos. Entre ella y la Mitjana, ligeramente excéntrica, encontramos la sierra del Reventón, inyección de materiales plásticos triásicos con un importante contenido en yeso; pero lo más relevante para este estudio van a ser los afloramientos de crestas cretácicas que aureolan la depresión, puesto que va a ser siempre en sus laderas donde encontremos las estructuras de combustión.

Las más antiguas de ellas pertenecen a las edades Valanginiense y Hauteriviense del Cretácico inferior, situadas al oeste de la sierra Mitjana y cerrando la zona conocida como Foia Casans; se presentan aquí en una facies flyschoide de calizas y margocalizas algo arenosas y con presencia en todos sus tramos de glauconita, nódulos de hierro y mica. Los niveles aptienses 
y albienses que afectan a nuestra área de estudio todavía en la Foia Casans comparten características con los algo más meridionales de les Índies y, en torno del Reventón, hacia el sur, con niveles de margas amarillentas o verdosas, micáceas y algunas calizas intercaladas, junto con facies ferruginosas de nódulos y mineralizaciones de hierro. Por su parte, todo el frente occidental de la depresión de Fontcalent, desde la sierra de les Àguiles hasta el espolón sobre el que se encuentra la población de El Rebolledo, queda encuadrado geológicamente en paquetes de la serie rítmica albiense superior $-\mathrm{y}$, por lo tanto, de características similares a lo descrito- que cortan la isócrona entre las edades Albiense y Cenomatiense, ya en el Cretácico superior (IGME, 1978, 14-24).

Dicho esto, hay que tener en cuenta que estamos trabajando en una escala 1:50.000 y, obviamente, los datos que se aportan se ajustan a ella. Es por esto por lo que consideramos baladí detenernos en afloramientos menores, como los jurásicos al norte del Reventón u otros cretácicos que rompen el glacis cuaternario del fondo de la depresión, o aun se intercalan en lentejones de diferente cronología o composición a las generalidades aportadas más arriba; del mismo modo, las estructuras que estudiamos se construyen sobre aportes cuaternarios en la base o el derrumbe de las crestas del Cretácico. En la parte contraria, la mención de determinado elemento en la Memoria del IGME constituye una prueba de su significación, y sobre esta base nos apoyaremos a la hora de buscar explicaciones funcionales a los hornos.

\section{NOTICIAS SOBRE LA PRESENCIA HUMANA}

Como venimos diciendo, estos hornos no aparecen en contextos poblacionales, ni siquiera en sus inmediaciones, lo que, sumado a la imposibilidad de una asignación cronológica en este punto del estudio, va a ensanchar el marco histórico hasta sus límites más amplios si no queremos obviar a ninguno de los posibles responsables de su construcción; será trabajo de la interpretación posterior no sólo reducir este margen, sino también entrar a considerar la necesidad de buscar una explicación en las comunidades inmediatas a las estructuras, como veremos. De este modo, creemos obligado referirnos a la secuencia ocupacional completa de una de las zonas de la comarca en la que ésta alcanza un grado de continuidad más perfecto. El límite territorial que manejaremos será las tres partidas rurales entre las que se distribuyen las estructuras de combustión, que en esencia se circunscribe a la unidad geográfica natural que ya hemos tratado y que equivale a suponer un radio máximo aproximado de unos 5 $\mathrm{km}$ desde las agrupaciones de hornos.

Según el inventario de yacimientos arqueológicos de la Direcció General de Patrimoni Cultural Valencià, la primera presencia atestiguada materialmente en la zona corresponde al Calcolítico, representada por los enterramientos de la Cova del Fum, en la vertiente oriental de Fontcalent, cuya naturaleza funeraria, precisamente, llevó a indicar la consiguientemente esperable presencia de contextos habitacionales en las proximidades todavía no documentados (Hernández, 1989, 52). J. Carbonell dirigió hacia 1963 un primer estudio de la cueva, pero quedó inédito hasta el trabajo de J. Soler (2002, 358-367), en el cual queda en el aire la posible existencia de niveles precerámicos de ocupación anteriores al uso funerario de la cueva. El siguiente hito lo encontramos en el poblado de la Edad del Bronce localizado en el Cerro de los Lobos, que ocupa un lugar central en la serie de elevaciones que se extienden desde la sierra de les Àguiles a la Mitjana, marcando el límite municipal con Agost; únicamente se ha publicado una somera descripción de las evidencias en superficie (López Seguí, 1996, 7982). En cualquier caso, de él destaca el control visual sobre el Camp d'Alacant, desde un punto que, a la vez, domina perfectamente el acceso al Vinalopó, a sólo 11 $\mathrm{km}$. Por lo demás, se ha señalado la particularidad de no contar con tierras aptas para el cultivo en su área inmediata, sino un poco más alejadas, pero sí afloramientos de arcillas rojas y yeso - ambos en explotación actualmente-.

Volviendo a las laderas de la Fontcalent, los últimos trabajos realizados sobre el yacimiento homónimo, en una elevación al noreste de la sierra, cerca del manantial termal y atravesado por el camino de l'Alcoraia, parecen apuntar a una redatación en la Edad del Bronce Final o el Orientalizante para la primera ocupación del sitio (Moratalla, 2005, 96-97). No sólo apunta en este sentido una reciente revisión (Ximénez de Embún, 2007, 139-145) de parte de los materiales que se pensaban pertenecientes a la Tardoantigüedad (Reynolds, 1985, 257-258 y 262), sino que la última intervención arqueológica, llevada a cabo a raíz de las obras del AVE, así lo confirma ${ }^{3}$. De esta forma, encontramos una secuencia ocupacional en esta parte de Fontcalent que abarcaría de los últimos momentos de la Edad del Bronce hasta época emiral (Gutiérrez, 1986,153), con algunos lapsos de abandono, como entre los ss. IV y II a. C., o en el V d. C. Por tanto, en este sitio arqueológico tenemos la primera mención a la Edad del Hierro y al horizonte ibérico, del cual se han recuperado materiales y algunas estructuras en los citados trabajos; salvando éstos, la única excavación arqueológica en el sitio fue una campaña dirigida por E. Llobregat en 1971, que puso al descubierto algunos muros sin llegar a alcanzar el objetivo que se había propuesto de contextualizar un fragmento de cerámica inscrita documentado durante una prospección por $\mathrm{V}$. Bernabeu (Llobregat, 1977, 23). Pero, sin duda, la pieza más conocida del conjunto es el citado fragmento

\footnotetext{
3. Información proporcionada por el Prof. Jesús Moratalla, desde la empresa Arquealia, como co-director de dicha intervención.
} 
inscrito en cursiva visigótica, fechado a través de la analogía con el alfabeto empleado en las pizarras esgrafiadas abulenses en el s. VI (Llobregat, 1970, 195).

Prácticamente en el extremo contrario de la zona que nos ocupa, en un cerro entre la Casa de l'Alcoraia y la sierra de les Àguiles, las obras de construcción de un embalse exhumaron una buena cantidad de cerámica, obligando a realizar una excavación de urgencia (Uroz, 1985). El objetivo de esta intervención fue dilucidar hasta qué punto las obras ponían en peligro el sitio arqueológico, cuya existencia se podía suponer, $\mathrm{y}$, tras comprobar que no se detectaban estructuras en la zona de afección, aislar la extensión del mismo. A pesar de los escasos resultados, fruto, en gran parte, de la remoción previa de tierras por los trabajos de agricultura en la parte más llana y de clandestinos en el cerro, se documentaron varios muros de una instalación romana. El conjunto material asociado apunta a una ocupación ibérica, sobre la cual continúa la romana sin interrupción, al contrario de lo que ocurre entre que ésta se abandona, aparentemente en el s. IV (Azuar et alii, 1990, 54), y se reanuda el registro en la Baja Edad Media, perfectamente caracterizado por el hallazgo de platos de Paterna y Manises de entre los ss. XV y XVII.

Sin embargo, la presencia humana en el contexto inmediato de este sitio pudiera entenderse a partir de la toponimia misma, pues no sólo la propia Alcoraia es inequívocamente de origen árabe, sino que también el cercano caserío del Campello, interpretado como un mozarabismo, ha dado pie a plantear unas raíces anteriores incluso al s. VIII para un topónimo que se mantendría durante el horizonte islámico (García, 1987). De este modo, tenemos que el núcleo de Fontcalent irá decreciendo hasta verse minimizado más allá del s. VIII (Azuar et alii, 1990, 43-54, 73-80; Ximénez de Embún, 2007, 161-162), mientras que l'Alcoraia pudo representar un punto destacado por su posición en la vía que penetra hacia el interior desde Alacant. Se ha sostenido, de hecho, que el propio topónimo islámico encuentre su origen en $a l$-Qaric $a$, o bien su diminutivo al-Qurayica, que vendría a traducirse como «centro de una calzada pequeña o desviación de otra que se considera principal» (García, 1987, 154), lo que ha valido para conectarla con los itinerarios altomedievales y la referencia a celeret, entendido primero como una mansio entre Aspis y Lucentum, que pudiera ser precisamente el nombre de la posterior Alcoraia o del poblado de Fontcalent (Llobregat, 1983, 238), y con relecturas posteriores, una vez cruzado con las variantes celeri y celeris, como una anotación para la existencia de un camino más rápido (Poveda, 1991, 69). En cualquier caso, el corredor de Agost, inmediatamente al norte de nuestra zona de estudio, es, sin duda, una buena zona para que transitara un vial en dirección a la costa, desgajado del que bajaba hacia Ilici desde el interior.

$\mathrm{Si}$, a partir de lo expuesto, vamos a encontrar en todo momento pequeñas explotaciones agrícolas o alquerías en uno u otro punto de estas partidas rurales, a partir de la Baja Edad Media y, sobre todo, ya durante la Edad Moderna, se ensanchará paulatinamente el área cultivada y la dispersión del poblamiento por la zona, de manera que en la primera mitad del s. XVIII ya encontramos los principales núcleos de población y casas señoriales, entre las que destaca la de los Bouligny ${ }^{4}$ por la extensión de tierras que aglutinará -prácticamente todas entre les Índies y la sierra Mitjana-, en buena parte dedicadas a la vid. Sin embargo, no hay una descripción de esta zona en concreto hasta el s. XIX, con el Diccionario de P. Madoz (1845-1850, I, 648), en el cual se anotan los cultivos desde Fontcalent y sus alrededores hasta la ciudad: algarrobos, higueras, cereales y barrilla, a los que vienen a sumarse en la vecina Sant Vicent el almendro y el olivo.

Nos interesa destacar aquí, por las implicaciones que va a tener en nuestro estudio, la referencia a la barrilla, que Madoz da como un cultivo propiamente dicho, mientras que de Bendicho, quien escribiera dos siglos antes y sólo de forma genérica para todo el término municipal, únicamente se puede deducir el aprovechamiento de un tipo de plantas que crecen naturalmente en amplias zonas de las cercanías de Alacant (Bendicho, 1650, 124-125). Precisamente en el tiempo que transcurre entre ambos autores va a conocer su apogeo la explotación y comercialización de la piedra de sosa, obtenida de la incineración de estas plantas, y no deja de ser significativo que Viravens $(1876,67)$ ya no la cite en su descripción de las partidas de Fontcalent, l'Alcoraia y El Rebolledo. En cualquier caso, parece ser que los Bouligny sí la cultivaron -0 , por lo menos, hicieron negocio con ella-durante la segunda mitad de la centuria de 1700 (Ribes, 1994-1995, 88).

Para el resto de actividades económicas desarrolladas en estas partidas empecemos por volver a Bendicho. Como decíamos, este autor apenas particulariza su descripción, pero sí menciona minas de «jaspes y pórfidos en los montes de Borrachina, San Francisco y sierra Mediana» (1650, 122-123), y, sin duda, parte de las minas de yeso que abundan en el término podrían bien entenderse en l'Alcoraia, atendiendo a las características geológicas ya descritas; para las tierras incultas advierte que en muchas partes existen bosques, y sin duda debieron de existir en estas partidas antes de imponerse la agricultura de secano en

4. En el Archivo Histórico Provincial de Alacant (Prot. 1657, fols. 248-154) se conserva el documento de compra de dicha casa por parte de Juan Bouligny y Maria Pared, el cual, fechado en 1745, nos da una referencia ante quem para su construcción. Sobre la ortografía del apellido -adaptación al francés del italiano Bolognini-, en este trabajo escribiremos Bouligny para hacer referencia a la familia, que se establece en la ciudad hacia 1712 proveniente de Marsella (cfr. Palencia, 2007); ésta es la primera forma atestiguada, y probablemente la que usaron para autodenominarse, por más que sea frecuente encontrarlos en los documentos como Bouligni, Boulini o Boliny. Las variantes Lo Boligni y El Bolini han quedado fijadas en la toponimia alicantina para la casa en l'Alcoraia y las tierras circundantes que pertenecieron a su finca, respectivamente, y en este sentido las usaremos. 
los siglos posteriores. Al listado de minerales explotados se viene a añadir el famoso intento de la mina de azogue de La Corneja, próxima a l'Alcoraia, referido tanto por Madoz (1845-1850, I, 648 y 653) como por Viravens $(1876,67)$ y, a la postre, del todo infructuoso, pues únicamente se obtuvieron mineralizaciones férricas.

Ya en el s. XX va a producirse una contracción de la superficie cultivada, la cual en su momento de máxima extensión abarca la práctica totalidad de las tierras susceptibles de aprovecharse siquiera mínimamente, con lo que de destrucción del paisaje anterior conlleva, de manera que actualmente abundan los bancales abandonados y los caminos más o menos deshechos en los alrededores de las sierras de Fontcalent y Mitjana, incluyendo las ruinas de varias casas y de la ermita de Sant Joan, una obra de finales del s. XVI o XVII, con amortización del material constructivo del sitio arqueológico de Fontcalent, y que otrora resultaba un hito para el llano que la rodea (Ximénez de Embún, 2007, 120-121). En su lugar, la mitad oriental de la Fontcalent y buena parte de los cerros que circundan la Mitjana quedan ocupados por canteras de diferentes materiales, uso industrial al que viene a sumarse la planta de compostaje en la solana de la sierra Mitjana y el polígono industrial de Les Atalaies, en expansión hacia la Fontcalent desde el sur. La otra mitad de las partidas, la occidental, ha experimentado en los últimos años cierto crecimiento demográfico en forma de casas de campo más o menos diseminadas, a partir de las vías de comunicación -CV-824 y carretera El Rebolledo-l'Alcoraia principalmente-.

\section{DESCRIPCIÓN DE LAS EVIDENCIAS}

Como no podía ser de otra forma dada su naturaleza constructiva, existe cierta variabilidad morfológica en las estructuras que estudiamos, pero este hecho no es óbice para poder aportar unas características-tipo a modo de definición genérica de los hornos con que nos vamos a encontrar, la cual será matizada en un segundo momento y a medida que vayamos describiendo una a una las evidencias registradas. Así, podemos entender que las estructuras de combustión localizadas en el entorno de la sierra de Fontcalent presentan una forma circular u ovoide, con un diámetro medio próximo al metro. Si bien la mayoría de ellas se ha erosionado horizontalmente, lo que provoca que se encuentren como círculos o planchas más o menos cóncavas dependiendo de la cota que haya alcanzado la pérdida de material de la estructura, uno de los ejemplares hallados se sitúa en el talud de una rambla y permite confirmar que, efectivamente, se trata de cubetas. Esta cubeta está formada por una capa de arcilla de unos 2 $\mathrm{cm}$ de grosor, endurecida por la acción del fuego y de una intensa tonalidad grisácea, que será la base de las mediciones que presentemos, aureolada en su exterior por una franja rubefactada cuya potencia oscila entre los 7 y los $12 \mathrm{~cm}$. Eventualmente, y siempre dependiendo de la forma en que se erosiona, presenta buen número de carboncillos en sus derrumbes, sin duda procedentes del interior de la solera, así como paquetes de tierra cenicienta. Por otra parte, es interesante añadir que el efecto del calor que se ha aplicado a estos paquetes de tierra les ha dado una dureza superior a la tierra que los rodea, y esto va a traducirse en una manifiesta erosión diferencial, al punto de que no sólo van a quedar resaltadas las soleras en el momento en que empieza a perderse la tierra que las rodea, sino que se puede llegar a diagnosticar la existencia de un horno por sus últimos restos, resultando útil fijarse en este factor, como veremos.

Todos los hornos que hemos estudiado se ubican en pendientes ligeras con una composición más bien arcillosa, de manera que resulta lógico pensar en un origen inmediato para el preparado del citado revestimiento interior de las estructuras. Con únicamente un ejemplar aparecido en solitario, se puede generalizar que se agrupan en zonas con una densidad muy variable, que va desde dos a siete o más estructuras. Dado que estas concentraciones de estructuras se encuentran siempre en las inmediaciones de los afloramientos cretácicos, se contabilizarán como pertenecientes a la misma zona los exponentes hallados en las laderas de una misma cresta; por lo que toca a las distancias, es significativo que en la práctica totalidad de grupos los hornos no se separen más que unos metros entre sí, superando sólo eventualmente los $10 \mathrm{~m}$, mientras que de una zona a otra encontramos un mínimo de $150 \mathrm{~m}$.

Esta sistematización encuentra una justificación añadida en las circunstancias particulares del registro, donde, a las dificultades de localización y contabilización propias de unas estructuras aisladas y de una entidad relativamente endeble, ostensiblemente complicadas de distinguir en el momento en que se espesa el matorral o dependiendo del grado de pérdida y aun de la luminosidad y otras condiciones del terreno, se suma el hecho de la incidencia antrópica en la transformación del paisaje que las rodea. Esto equivale a decir que no sólo somos conscientes de que algunas estructuras han quedado fuera del registro o únicamente podemos incluirlas en condiciones ciertamente precarias, sino que el hecho de aparecer agrupadas en las laderas incultas y separadas por campos, edificaciones y caminos no nos está asegurando que no pudieran originalmente haberse encontrado hornos más alejados de las estrictas elevaciones, y que sean éstos los testigos que han quedado en áreas residuales que no se han podido aprovechar posteriormente para la agricultura.

En este sentido, de lo recogido de los informantes que habían trabajado en la contornada, destaca la descripción de dos de las zonas que no hemos podido localizar durante el estudio: una, con dos o tres hornos seccionados por las obras de ensanche del camino El Rebolledo-l'Alcoraia en el momento de asfaltarse, que los habían dejado en el talud de uno de los bancales 


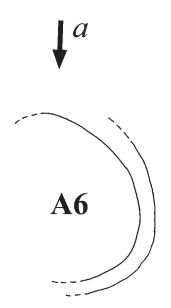

\section{$\mathrm{N}$

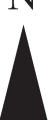
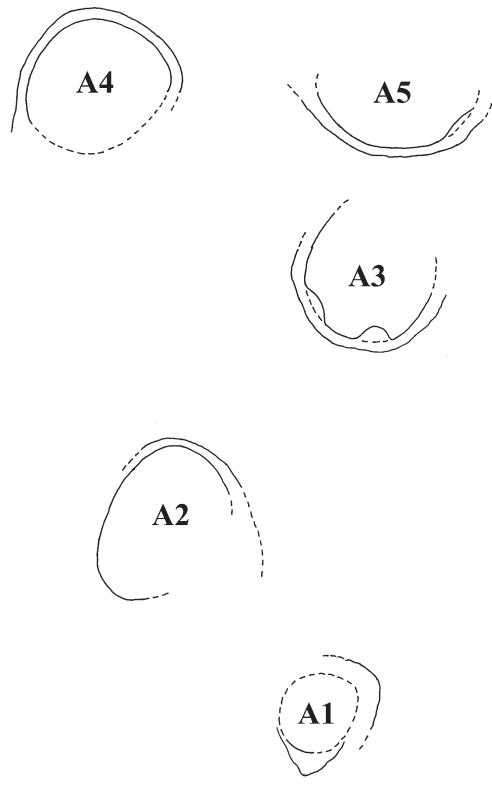

$\uparrow_{b}$

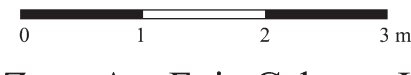

\section{Zona A - Foia Cabrera I}

Figura 2: Planta del conjunto de estructuras de la zona A tal y como aparecen en superficie; la línea interior corresponde a la solera, mientras que la exterior corresponde al perímetro de afección de la rubefacción. unos 40 o $50 \mathrm{~cm}$ por debajo de la cota de uso agrícola; la segunda, en los bancales de olivos inmediatamente el oeste de la Fontcalent, entre la carretera que se dirige a l'Alcoraia desde la salida de la autovía y el Barranc de l'Infern, en la que las avenidas de agua habían acabado por retirar la tierra labrada para dejar al descubierto tanto las raíces de los árboles como algunas estructuras de las que nos ocupan. Por desgracia, puesto que no son noticias contrastadas, su relevancia para este trabajo queda minimizada.

\section{ZONA A. FOIA CABRERA I (UTM: 707648-4248428)}

La Foia Cabrera está delimitada meridionalmente por el Barranc de l'Infern cuando le quedan unos $4 \mathrm{~km}$ antes de llegar a los pies de la Fontcalent, al este-sureste, en un paisaje marcado por la alternancia de cultivos de secano, como almendros y vid recientemente planta$\mathrm{da}$, con pequeñas elevaciones yermas y salpicado de construcciones residenciales, si bien con una densidad

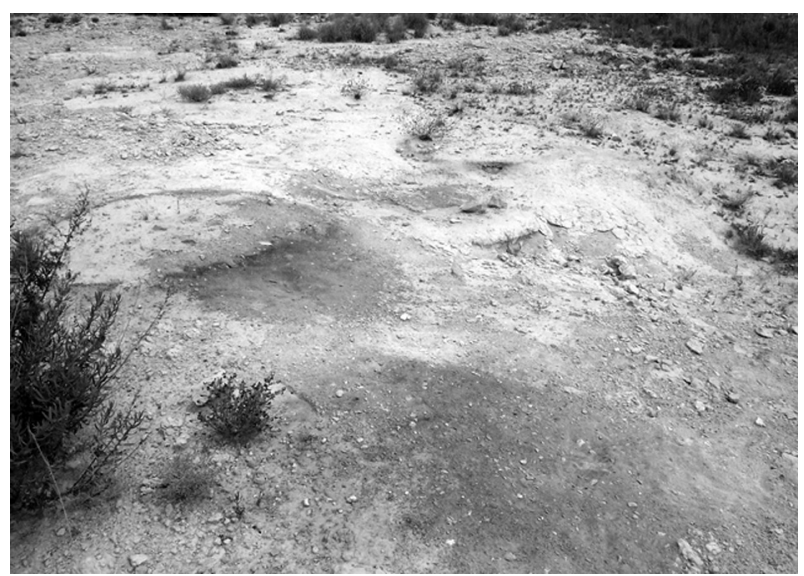

Figura 3: Vista general de la zona A desde el sur el 28 de julio de 2007; en primer término, A1, prácticamente desaparecido; tras él, A2 y A3, a la derecha.

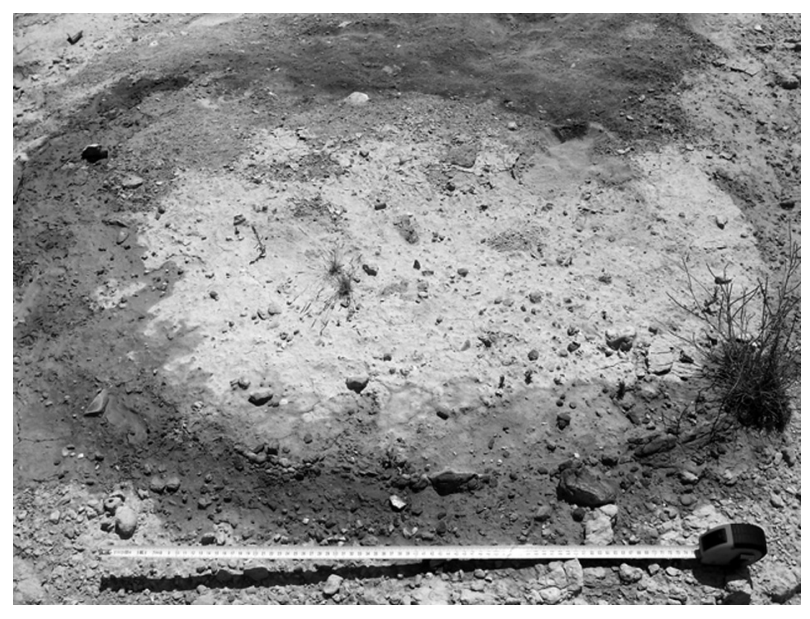

Figura 4: La estructura A2 (21/VII/07), tomada un año antes de la redacción del estudio: se aprecia el incipiente desmonte de la solera al fondo; el flexómetro marca $75 \mathrm{~cm}$. 


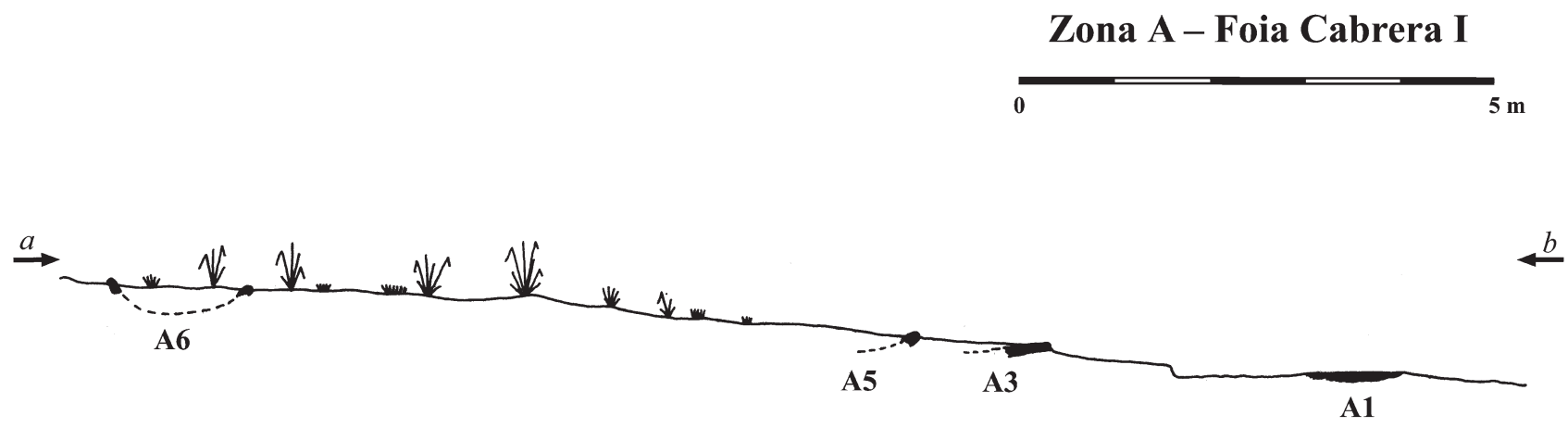

Figura 5: Sección de ladera de la zona A; se indica en línea discontínua el desarrollo hipotético de las soleras.

muy inferior a la constatada más al oriente, articulando los campos una red de caminos de los cuales en los últimos años se han empezado a asfaltar algunos. En el margen derecho de uno de los que permanece sin asfaltar, ramal que progresa hacia el oeste-noroeste desde el camino El Rebolledo-l'Alcoraia hasta la CV824 , encontramos la primera agrupación de estructuras de combustión de nuestro estudio. Sobre el camino, quizá sea interesante señalar que es utilizado como límite catastral entre los polígonos 026 y 029 , lo que $a$ priori lo dota de cierta antigüedad respecto de los que lo rodean.

El límite oeste de la parcela 77 (polígono 029) queda inculto por la presencia de una de las citadas crestas cretácicas, en cuya pendiente suave hacia el camino se disponen los hornos, de los cuales se ha podido constatar la presencia de siete con certitud, lo que la convierte en la zona con una concentración más elevada de estructuras (Figs. 2 y 3 ). A ellas habría que sumar tres manchas rojizas perfectamente destacadas -de hecho, responden al rascado superficial, deshaciéndose en tierra roja y no, como otras manchas menos definidas, en la amarilla propia de los sedimentos naturales-, al este de A3 y A5, que pudieran corresponder tanto a sendas estructuras ya perdidas hasta su base como a fuegos relacionados con otra actividad indeterminada. De los hornos tampoco se pueden dar medidas precisas de todos, pues muchos no presentan visible más que un tramo de la circunferencia. Por su parte, A1 ha quedado reducido a tierra suelta, por más que conserve parte de la forma y un diámetro de $75 \mathrm{~cm}$. Sobre él, A2 (Fig. 4) también presenta signos avanzados de erosión y su mitad meridional queda bastante desdibujada, dándole una forma ovoide posiblemente más exagerada de lo que tuvo en origen; así, su diámetro mayor alcanza los $130 \mathrm{~cm}$ mientras que el menor mide 100, pero la mancha rojiza a este lado, con una cota más baja, prácticamente cuadruplica los habituales $7-8 \mathrm{~cm}$ que se registran alrededor del resto de la circunferencia y este derrumbe vale para imaginarle una forma algo más compensada. A su norte-noreste queda A3; presenta un diámetro de $105 \mathrm{~cm}$ y, aunque muestra un buen trozo de la plancha cenicienta, su circunferencia queda cubierta parcialmente al norte, donde muy próximo, si no superpuesto, encontramos A5, del cual no podemos más que constatar la existencia al no permitir el perímetro visible una medición del diámetro. Al oeste, A4 es el que hoy en día presenta unas características más claras, con un diámetro de $96 \mathrm{~cm}$ prácticamente visible en su totalidad y una potencia de rubefacción de 8 $\mathrm{cm}$, claramente delimitada por el surco que la afección diferencial de la erosión ha generado a su alrededor. Cierran el grupo otras dos estructuras al norte-noroeste: A7, insinuada muy débilmente entre el matorral, y A6, en la cota más alta del grupo (Fig. 5), con un diámetro de $100 \mathrm{~cm}$ y una rubefacción de $10 \mathrm{~cm}$.

Prácticamente todo el material de origen antrópico que se registra en superficie se puede explicar por el derrumbe de una casa situada en la cima de la elevación y actualmente arrasada hasta unos $20-30 \mathrm{~cm}$ del suelo. Encontramos ceràmica d'aigua típica de la zona y diferentes restos de vidriados en verde, ámbar y rojo, junto con fragmentos mucho más escasos de loza bícroma e incluso una base de taza que recuerda a los diseños de La Cartuja. Vienen a sumarse puntualmente alguna lata y varios contenedores plásticos, especialmente en el margen contrario del camino y sin duda relacionados con los trabajos en los bancales que circundan la zona A; pero quizá el material más abundante sobre los hornos sean restos de tejas de tipo moruno.

\section{ZONA B. FOIA CABRERA II (UTM: 707490-4248488)}

Siguiendo el camino en dirección oeste-noroeste poco más de $150 \mathrm{~m}$, se alcanza en el margen izquierdo una serie de crestas que, con sus 208 m s.n.m., superan en entidad a las anteriores y deben de ser las últimas estribaciones de la línea que pone límite septentrional a los parajes del Desert y el Carme, de la que quedan separadas por el Barranc de l'Infern. Antes del arranque de la pendiente que escala el cerro, se extiende una lengua de tierra paralela al camino, con matorral bastante tupido pero que en su tramo más occidental aparece libre de vegetación, y donde, además, y sin duda por la acción recurrente de las torrenteras, se ha dibujado una especie de amplio vado a la misma cota que el camino. 
Queda toda la superficie que nos interesa ahora comprendida en la parcela 59 del polígono 026.

La estructura $\mathrm{B} 1$, más próxima al camino y por tanto más afectada por la erosión, presenta la plancha cenicienta informe y enrasada al fondo de la solera, con apenas unos $50 \mathrm{~cm}$ conservados en su parte más amplia; sin embargo, se puede ensayar la medición del diámetro original por los $90 \mathrm{~cm}$ que alcanza el vaciado que ha quedado a su alrededor, con la pérdida del resto de la tierra rubefactada. Por su parte, B2 queda sobre el margen de la línea de erosión que forma el escalón del vado, a una distancia de 5,90 m de B1; tampoco muestra la circunferencia completa por permanecer una parte aún oculta bajo la tierra de dicho escalón, y el perímetro visible está claramente afectado por la acción del agua, de manera que ha perdido en parte su forma circular, pero mantiene un diámetro mayor de $90 \mathrm{~cm}$ y unos $10 \mathrm{~cm}$ de rubefacción en torno a sí. Como en la zona A, también aquí encontramos manchas rojas en las cercanías que no llegamos a poder catalogar como estructuras análogas a las que estudiamos: una excéntrica en parte sobre B2, que al rascado superficial parece deshacerse en tonos negruzcos, pero sin delatar una forma tan definida como las soleras, y otra al oeste, ya entre la maleza. El registro de materiales humanos es aquí muy parco, y apenas se han podido contar un par de fragmentos informes de cerámica común.

La zona B se completa con una tercera estructura apartada del resto del grupo unos $50 \mathrm{~m}$ al sur pero sin llegar a abandonar la parcela 59. B3 se encuentra en el borde de la pequeña rambla que discurre desde la cima del cerro hacia el bancal, remontando algo más de 40 $\mathrm{m}$ desde éste $\mathrm{y}$, por tanto, relativamente alejada del camino. Presenta una forma ciertamente desdibujada que con toda probabilidad ha de responder a la erosión: aproximadamente cuadrangular, de más de 100 por $45 \mathrm{~cm}$, uno de sus lados cortos se pierde entre la maleza mientras que otro aparece redondeado; al quedar a diferentes cotas por la acción del agua, tampoco sus lados largos son iguales, y mientras que en uno se aprecia la aureola de rubefacción sin apenas muestras de la cubeta arcillosa, en el desmonte sí aparece una capa grisácea con presencia de carboncillos. Con esta descripción es evidente que no podemos incluirla con las garantías necesarias bajo las características generales del resto de estructuras de combustión, aunque tampoco se puede descartar una homogeneidad con el resto del grupo perdida merced a la acción erosiva. Sobre los restos de artefactos, en esta parte de la elevación son inexistentes.

\section{ZONA C. LES ÍNDIES (UTM: 708699-4249540)}

El cerro del Reventón acaba al norte en una serie de lomas conocidas como les Índies, justo antes de abrirse nuevamente a los campos de cultivo plantados de olivo de la antigua heredad de los Bouligny, de quienes toma el nombre el paraje que se extiende desde aquí hasta la sierra Mitjana y en esta parte queda dentro de una parcela de grandes dimensiones, la 94 del polígono 030. Equidistante de ambas elevaciones progresaba el camino viejo de l'Alcoraia, hoy distorsionado por el trazado del AVE, que se le superpone en un buen trecho; éste pasa junto a la casa de Lo Boligni, y de aquí parte un camino de menor entidad en dirección sur hacia les Índies para bordearlas en paralelo a la plataforma ferroviaria antes de torcer al norte y reintegrarse al camino principal. La tercera agrupación de hornos aparece en un pequeño espolón a la margen izquierda del camino, dejando les Índies a la derecha; por tanto, a ambos lados encuentra bancales, pero no hay ni construcciones actuales ni señales de cuidados agrícolas recientes.

Las dos estructuras que componen la zona $\mathrm{C}$ se caracterizan por un nivel de pérdida aparentemente inferior al registrado en los grupos de la Foia Cabrera y, por lo tanto, su visibilidad no va a ser tan obvia como la de aquéllos. En este caso, a C1 se le intuye una forma bastante circular, de $115 \mathrm{~cm}$ de diámetro, pero no se verifica la costra cenicienta de la cubeta en todo su perímetro $\mathrm{y}$, cuando lo hace, aparece mucho más vertical, lo que sin duda debe explicarse por encontrarnos en una cota más alta de la estructura que los casos ya descritos. Unos $4 \mathrm{~m}$ más al occidente y directamente sobre el borde del espolón se localiza C2 (Fig. 6), que repite la misma casuística y únicamente permite medir su diámetro, unos $100 \mathrm{~cm}$, a partir de la aureola de rubefacción de $6 \mathrm{~cm}$ y no de los escasos tramos de arcilla endurecida visibles; de él hay que añadir los varios centímetros de diferencia entre su cota superior e inferior, ya prácticamente a ras del camino, por las consecuencias que de esta circunstancia puedan derivarse en las mediciones.

En lo tocante al material en superficie, se ha registrado la presencia relativamente abundante de fragmentos informes de cerámica de pasta tosca y oscura,

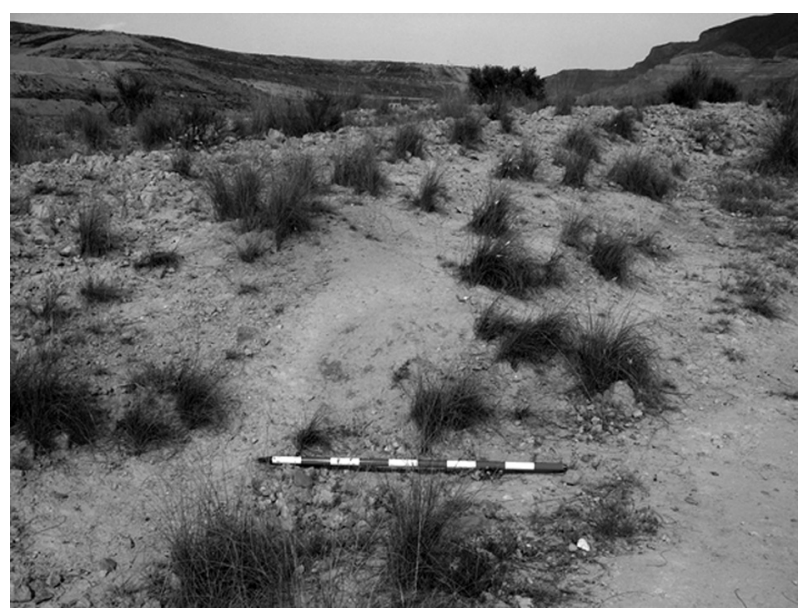

Figura 6: Vista general de la zona C el 22 de junio de 2009, con el camino parcialmente visible a la derecha; en primer plano, $\mathrm{C} 2$; más alejado se intuye $\mathrm{C}$, ligeramente excéntrico. 
difícil de catalogar, como de vidriados que no escapan al repertorio tradicional alicantino de los últimos siglos, pero junto a ellos se ha localizado también un fragmento de sigillata, probablemente hispánica, si bien no se ha abundando en su caracterización al ser la única zona que presenta restos de este tipo. En cualquier caso, ninguno de ellos aparecía directamente sobre los hornos, o en sus derrumbes o cercanías, sino dispersos por los bancales circundantes, lo que redunda en una difícil vinculación entre las estructuras de combustión y la cerámica, que posiblemente ha de explicarse en su mayoría por acciones y momentos diferentes al uso de los hornos.

\section{ZONA D. FOIA CASANS I (UTM: 708659-4250314)}

La Foia Casans es una pequeña depresión de tendencia triangular inmediatamente al oeste de la sierra Mitjana; al sur se abre hacia El Bolini en una pendiente cada vez más suave, de manera que desagua en el sistema de Fontcalent, de quien es prácticamente la cabecera colectora septentrional. Esta posición ha modelado el paisaje de la foia de manera que se alternan crestas de calizas y, sobre todo, margocalizas cretácicas con torrenteras, ya sean tan acusadas como la rambla que discurre a los pies de la Mitjana o ya simplemente taludes erosionados por el agua aprovechando los caminos que la articulan. Entre ellos se encuentra el citado camino viejo a l'Alcoraia, que se endereza hacia el norte antes de llegar a Lo Boligni buscando el punto de fuga que resulta la cabecera de la Foia Casans. La única estructura de la zona $\mathrm{D}$ se encuentra en uno de estos taludes, de algo más de un metro de altura desde la cota del camino que hace las veces de separación entre las parcelas 80 y 81 del polígono 030, recayendo en esta última. A pesar de que hay restos en la práctica totalidad del suelo hábil de la Foia Casans de una actividad agrícola pasada, centrada en el olivar, la mayoría de los bancales están hoy en día abandonados y muy degradados.

A efectos de registro, el horno D1 presenta unas características de erosión que lo dotan de una importancia añadida, pues, mientras que comúnmente la pérdida de material se verifica sobre el plano horizontal, en este caso lo hace en el vertical (Figs. 7 y 8); asimismo, poniéndolo en relación con los otros exponentes descritos, se puede inferir que en el momento de su construcción, en lugar del talud formado por las avenidas de agua, debió de haber una pendiente relativamente ligera sobre la que se excavaría la estructura que, teniendo en cuenta el nivel de tierra arrastrado con posterioridad a ese momento, no tenía por qué ser única en la zona. De hecho, el nivel de pérdida de la estructura es bastante acusado y apenas conserva en el plano unas medidas máximas de 47 por $27 \mathrm{~cm}$, que no parecen alcanzar la mitad de la circunferencia original, mientras que la rubefacción presenta una potencia de $10 \mathrm{~cm}$ lateralmente y $12 \mathrm{~cm}$ bajo la solera, antes de

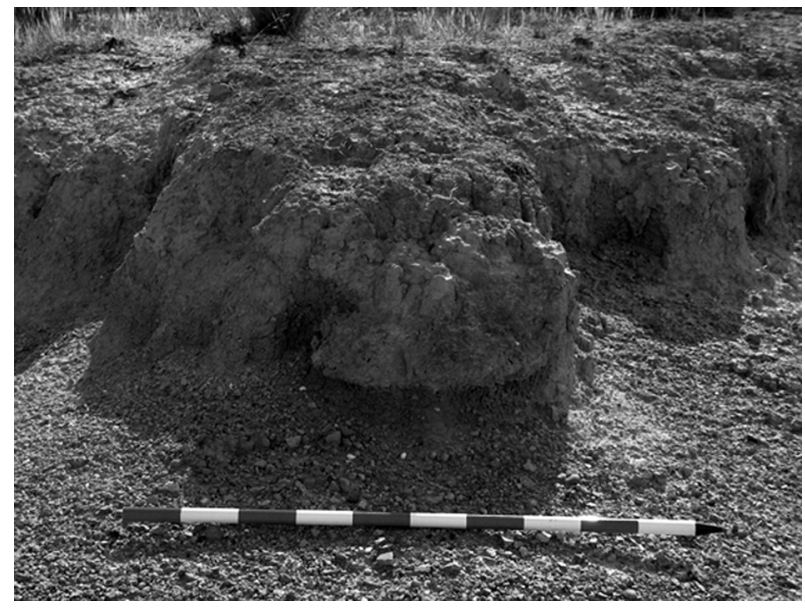

Figura 7: Estructura D1 erosionada verticalmente (22/VI/09); nótese el paquete de tierra cenicienta que rellena la solera con una altura algo superior a $10 \mathrm{~cm}$.

iniciarse el desmonte más suave del final del talud. En el interior del horno, hasta unos $10 \mathrm{~cm}$ por encima de su punto más bajo -y por tanto sin alcanzar la parte más alta de las paredes-, se detecta claramente un paquete de tierra mucho más oscura, con algún carboncillo embebido. En este sentido, creemos que es importante destacar que el nivel del suelo en la parte alta del talud queda todavía a algo más de $30 \mathrm{~cm}$ de la parte visible más alta de la solera, lo que se corresponde con las informaciones sobre otros hornos no documentados por debajo del nivel de uso agrícola ya mencionados; de aceptar, como parece lógico, que dicha potencia estratigráfica ha venido a añadirse sobre la cota de suelo en el momento de uso de las estructuras, habría que entrar a valorarlo como una seña de antigüedad relativa, teniendo en cuenta que la tendencia observada en todas nuestras zonas de estudio es precisamente la inversa, degradando las capas de tierra arcillosa en las torrenteras y no aportándoles sedimentos.

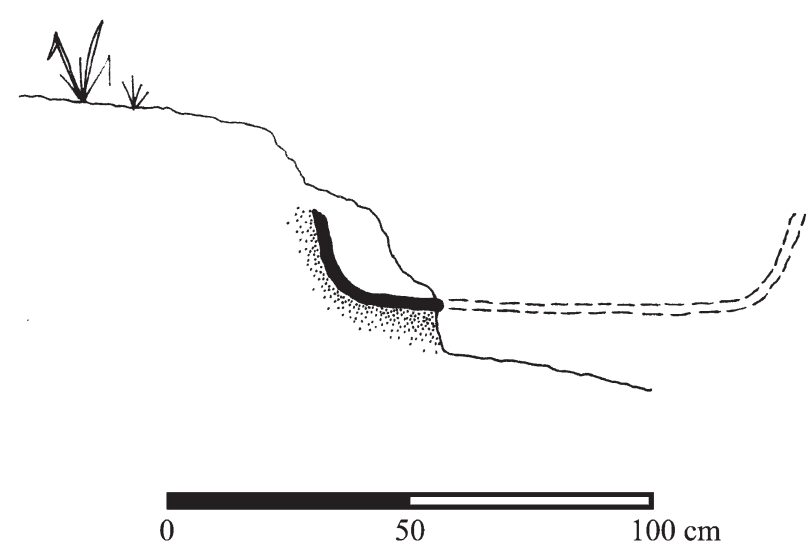

Estructura D1 - Foia Casans I

Figura 8: Sección del horno 1 de la zona D, según es visible en superficie; el punteado representa la zona rubefactada, y la línea discontinua la reconstrucción hipotética de la solera. 
Cerrando la descripción con los materiales en superficie, en este caso hay que contar con la proximidad de la zona de crecimiento residencial que arranca a poco más de $100 \mathrm{~m}$ al norte de D1, tras la elevación, y se prolonga por todo el último tramo del camino de l'Alcoraia antes de alcanzar la CV-824, a lo que hay que sumar las obras del AVE hacia el sur. De esta forma, abundan en los márgenes del camino depósitos de materiales de construcción actuales y otras basuras. También hay restos de contenedores cerámicos, pero en las inmediaciones del horno son bastante escasos y hay que alejarse varias decenas de metros al este para encontrar concentraciones en los bancales contiguos, en el centro de la depresión, con exponentes de los tradicionales tipos de ceràmica d'aigua y de foc, así como otros vidriados monocromos comunes.

\section{ZONA E. FoIA CASANS II (UTM: 708825-4250226)}

Superados estos bancales, encontramos otra serie de elevaciones no tan continuas y de una altura algo inferior a la de las que cierran la foia occidentalmente. Entre ellas discurre el camino viejo de l'Alcoraia en un tramo de $400 \mathrm{~m}$ prácticamente alineado norte-sur antes de torcer al norte-noroeste hacia la Casa dels Garcia y el final de la Foia Casans. La zona E, por tanto, está prácticamente rodeada de afloramientos cretácicos, con la única excepción de una serie de bancales totalmente abandonados que se abren orientalmente hacia la rambla que baja bordeando la sierra Mitjana. Como en el camino junto a D1, también aquí hay señales de erosión fruto de las avenidas de agua, y cuyo resultado ha sido la formación de un extenso vado en el margen occidental del camino, que es a la vez el límite entre los polígonos catastrales 030 y 021 y las parcelas 80 de aquél y 122 de éste, entre las cuales se reparten las estructuras de la zona E, a unos 195 m este-sureste de la D.

El horno E1 es el más visible (Fig. 9), directamente sobre el margen oriental del camino, y con un diámetro mayor de $110 \mathrm{~cm}$ y uno menor de $70 \mathrm{~cm}$, de forma que aparentemente era ovalado, por más que se pueda sostener una exageración de esta forma a causa del derumbe de la estructura volcado hacia el norte, donde, precisamente, se acumulan carboncillos y se pierde o desdibuja la capa de arcilla endurecida de la cubeta; al lado contrario, sin embargo, queda descubierta, y puede medirse una aureola de rubefacción en torno a ella de $8 \mathrm{~cm}$. Esta misma potencia de tierra roja afectada por el calor la encontramos en una estructura contigua, E3, 3'20 $\mathrm{m}$ al sur de la primera y parcialmente bajo el camino, por más que no acabe de ser un dato cronológico determinante al poderse haber ensanchado la pista con el transcurso de los siglos. Las características de E3 escapan a la pauta, pues no sólo presenta una silueta difícil de definir, sino que es la única en la que aparece una fina película blanquecina en algunas partes de la cara interior del perímetro, del cual una de sus paredes es recta en un tramo de unos $80 \mathrm{~cm}$. El hecho de verificar la típica tendencia circular en otras partes visibles de la estructura, con un diámetro de $110 \mathrm{~cm}$ -lo cual también encaja con el resto de hornos documentados-, y la aparición hacia el desnivel del margen del camino de otro tramo que aparentemente parece continuar desde un punto próximo a donde se pierde el primero, pero con una circunferencia no coincidente y sin la película blanquecina, puede estar remitiéndonos a una superposición de estructuras, quizá incluso con una reutilización posterior a su uso como horno que enluciera un lado aplanado, pero las posibilidades sin una limpieza superficial que ponga al descubierto los tramos aún enterrados son meramente especulativas; por esta razón, y una vez explicada la singularidad de

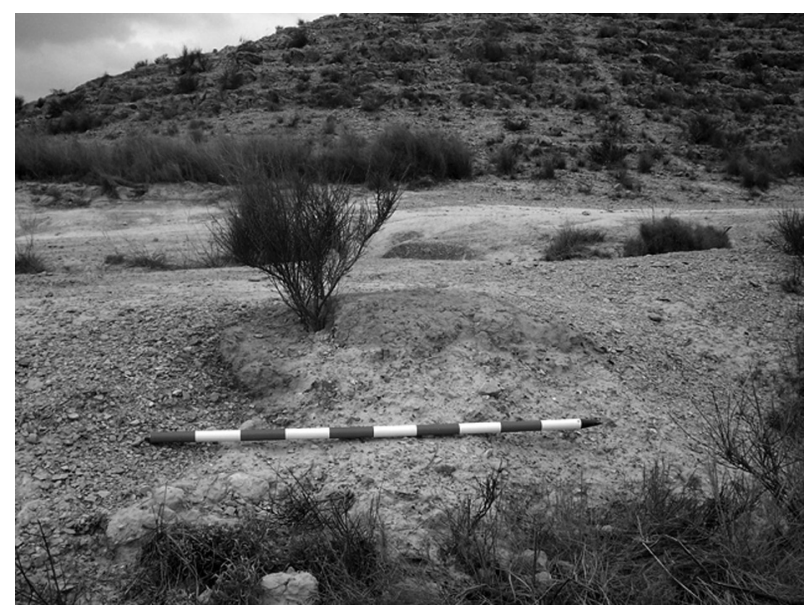

Figura 9: Restos del horno E1 el 22 de marzo de 2009, con el camino visible en segundo plano; nótese la afección diferencial de la erosión por la dureza de la tierra dentro del horno.
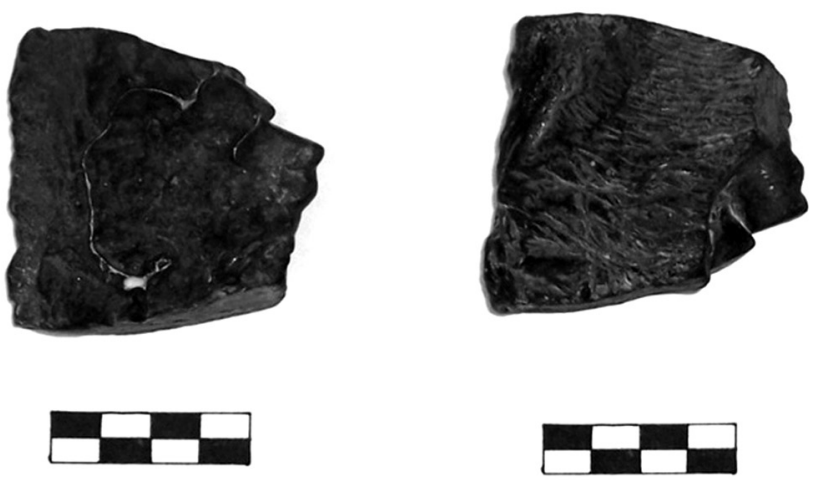

Figura 10: Escoria globular de Foia Casans II; la cara dorsal (izquierda) muestra la característica superficie lisa a chorretones, mientras que en la ventral (derecha) se aprecia el negativo del suelo por el que fluyó. 
E3, nos abstendremos de numerarlo como dos hornos tanto como de suponerle una normalidad que moviera a modificar la descripción general.

Por su parte, unos $30 \mathrm{~m}$ al sur de este grupo, al lado contrario del camino y por tanto ya dentro del vado descrito, hallamos unos restos muy dañados, prácticamente sólo identificables por la afección diferencial de la erosión en un diámetro de $60 \mathrm{~cm}$ sobre una mancha rubefactada que ha perdido la definición clara de su contorno, pero que deja ver una capa cenicienta de idénticas características a las cubetas deshechas de otras estructuras, mezclada con tierra del característico tono rojo intenso, según se rasca superficialmente, de modo que parece lógico interpretar que nos encontramos ante un último horno, cuya numeración será E2.

Desde su posición, E1 y E3 vierten hacia un abancalamiento muy degradado que apenas conserva un par de tocones entre el matorral más o menos denso. A pocos metros del horno en esta parte, cerca del límite septentrional del bancal, apareció semienterrada una escoria de reducción de hierro (Fig. 10), de la que trataremos en detalle en el apartado siguiente. En lo tocante al resto de materiales de origen antrópico, más allá de la escoria, carecemos aquí de toda señal en las inmediaciones de las estructuras, al igual que en la zona B, si bien avanzando hacia la rambla se ha encontrado puntualmente algún fragmento de ceràmica d'aigua.

\section{HACIA UNA INTERPRETACIÓN FUNCIONAL}

Si algo es sobresaliente en la descripción que hemos dado es la falta de un registro material antrópico recurrente que ayude a enmarcar nuestras estructuras en un proceso productivo dado o, siquiera, en una cronología que permitiera comenzar a descartar usos. Para el primer caso, carecemos de subproductos o residuos productivos en una escala significativa. Para el segundo, el material cerámico no parece vincularse necesariamente a los hornos en los casos en que aparece, y no existen diferencias sustanciales entre lo que se documenta en el entorno de los hornos y los volúmenes y variedad cerámica de zonas más alejadas. De esta manera, se le puede presuponer un origen deposicional desvinculado de ellos, merced a la presencia continuada de humanos en la contornada y especialmente con la extensión de la agricultura a la práctica totalidad del suelo aprovechable de estas partidas en los últimos siglos, antes que tratar de discernir sin mayores apoyos cuáles hay que entender vinculados y cuáles no.

Esta circunstancia se debe a la combinación de dos factores que juegan en nuestra contra: la propia naturaleza de la actividad económica de que son fruto las estructuras, y la incidencia de los procesos postdeposicionales y las dinámicas erosivas de la zona. Cualquier explicación ha de tener en cuenta ambos. Sin embargo, mientras que para la caracterización productiva podemos apoyarnos positivamente en las evidencias documentadas, aunque sean escasas -desde condicionantes como la localización al margen de los núcleos habitacionales y dispersos por el campo, señal casi inequívoca de que se está primando la cercanía a algún o algunos recursos que habrá que identificar, posiblemente, y en primera instancia, con el que se está transformando, hasta la propia morfología de los restos, la cual va a permitirnos descartar procesos para los que conocemos la estructura resultante o sabemos de características que han de darse necesariamente-, tanto la dinámica erosiva natural como otros procesos postdeposicionales que se puedan haber dado eventualmente nos van a permitir únicamente ideas en negativo. Sabemos que son potencialmente acusados y es lógico pensarlos un agente capaz de eliminar muchos de los restos susceptibles de apoyar una caracterización funcional de los hornos, pero carecemos de estudios que los cuantifiquen.

A partir de lo expuesto, podemos afirmar que tanto el enlucido arcilloso que recubre las cubetas como los valores que alcanza la rubefacción de la tierra a su alrededor nos están hablando de un proceso en el que se alcanzan temperaturas muy elevadas, lo que ya está eliminando la inmensa mayoría de actividades de combustión de materias vegetales, que en esta zona prácticamente se podrían reducir a la quema a cielo abierto sea de broza sea con cualquier otro fin, incapaz de afectar de tal modo a la tierra, o la eventual reducción de carbón, en unas estructuras morfológicamente muy diferentes. Y, por lo que toca a los minerales susceptibles de ser explotados, ni el alabastro ni la arcilla requieren de procesos que pudieran generar este tipo de estructuras, y los hornos de yeso y caleras, fácilmente documentables en muchos puntos de la comarca ${ }^{5}$, producen unas evidencias muy características que no se ajustan a nuestro caso. De este modo, y en función de una primera confrontación entre las posibilidades económicas de las partidas noroccidentales del Camp d'Alacant y la analogía morfológica de las estructuras por ellas producidas, podemos limitar en este punto a dos las actividades potencialmente capaces de explicar nuestros hornos, por más que el desarrollo de ambas hipótesis, con la metodología posible en este trabajo, no alcance un grado de determinación tal que permita la identificación sin reservas, como veremos.

\section{¿HORNOS PARA LA FUSIÓN DE BARRILLA?}

Se ha explicado en la contextualización histórica cómo durante la segunda mitad del s. XVIII se tiene constancia del cultivo de barrilla en las proximidades de la

\footnotetext{
5. Sin ir más lejos, en les Índies se conservan a bocamina, en la de yeso a cielo abierto que penetra ya en El Reventón, las ruinas de una instalación con tres hornos circulares de mampostería; también se han documentado algunas caleras en estas partidas, como la que fue destruida por las obras del AVE tras su registro arqueológico en 2006.
} 
Fontcalent. Éste es el momento de máxima expansión de tal actividad, irradiándose el método alicantino de la siembra, cultivo, quema y comercialización de la barrilla incluso a las Islas Canarias (Miralles, 1985, 77 y ss.), pero en principio no habría problemas para suponerle una fecha algo anterior, quizá en la segunda mitad de la centuria de 1600 , y con el tremendo despegue comercial que va a mutar en cultivo de exportación lo que por lo menos desde época islámica era un aprovechamiento complementario de plantas que crecían naturalmente en vastas zonas de las cercanías de Alacant (Azuar et alii, 1990, 87, 324-351), pues recordemos que en 1650 Bendicho sólo habla de su recolección y transformación en piedra. No en vano, el término barrella está documentado como topónimo desde el s. XIII, lo que ha valido de fundamento a la hora de asignarle un origen mozárabe (Sala Caja, 2003, 70-73), que en la zona del Ebro quedaba restringido a la Salsola kali mientras que en el País Valenciano servía para nombrar a otras especies del género Salsola o a la Halogeton sativus, vulgarmente barrella fina en nuestras comarcas, o de Alicante allí donde se exportaba. Las piedras obtenidas a partir de esta última eran las más valoradas, en función del alto porcentaje de sales que la planta es capaz de contener y la resultante cantidad de carbonato sódico $\left(\mathrm{Na}_{2} \mathrm{CO}_{3}\right)$ que se extrae en su incineración (Fernández, 1998), y a la postre elevó la ciudad a verdadero punto de referencia para la producción de sosa, captando su puerto incluso la de zonas tan distantes como Lorca (Velasco, 2004, 155).

Sin embargo, no hay una correspondencia entre la importancia socioeconómica de la barrilla y la publicación de estudios que la tengan como objeto, de los que hay que destacar los de Gil Olcina (1975) o Fernández Pérez $(1990,1998)$. Esta brecha es especialmente significativa en el caso de la arqueología, que ha de identificar los restos materiales de ese proceso productivo. En este sentido, únicamente contamos con una hipótesis de F. Sala Sellés ${ }^{6}$ a propósito, precisamente, del único referente a estructuras similares a las de Fontcalent, localizado durante la campaña de excavación de 2001 en el poblado ibérico de El Oral. Aquí aparecieron sobre el estrato de colmatación ibérico seis hornos que se construyeron una vez estaba el poblado abandonado, aunque todavía eran visibles los zócalos de los muros, próximos a los cuales se ubican, y la falta de materiales impidió tanto calcular el lapso de tiempo transcurrido entre uno y otro hecho como asignarles un uso (Abad et alii, 2001, 98-100);

6. La referencia ha de aparecer en las actas del Col-loqui internacional: El paisatge periurbà en la Protohistòria $i$ l'Antiguitat a la Mediterrània Occidental, organizado por el Instituto Catalán de Arqueología Clásica en Tarragona del 6 al 8 de mayo de 2009, con el título «Los espacios periurbanos en el área ibérica contestana: las novedades y algunas reflexiones históricas». uso que, a decir de Sala Sellés, podría ser el de nuestra primera hipótesis.

Así las cosas, el principal escollo que vamos a encontrar para desarrollarla va a ser la ausencia tanto de referentes materiales previamente identificados con este proceso productivo, como la escasez de fuentes históricas que nos informen de él con detalle, las cuales se reducen prácticamente al artículo de Mariano Lagasca publicado en $1818^{7}$, base, a su vez, de las descripciones técnicas vertidas en los trabajos de Fernández.

Tampoco existen certezas sobre el origen de esta práctica, pues si, por un lado, se apunta una cronología medieval (Sala Caja, 2003, 69) para la sustitución de la «sosa natural» por la obtenida de plantas saladas merced al colapso del comercio de natrón egipcio o, en cualquier caso, vinculado con la expansión islámica (Fernández, 1998), por el otro el uso de ciertas cenizas vegetales en la elaboración de lejías es consabidamente muy anterior. Y aquí conviene comenzar a distinguir entre la simple incineración a cielo abierto que se vino practicando hasta hace no tantos años en el marco de las economías domésticas para obtener jabón, incapaz de generar los restos documentados en las partidas de Fontcalent, l'Alcoraia y El Rebolledo, y el proceso técnico de fusión y transformación a estado sólido que periclitó a inicios del s. XIX.

El paso de uno a otro no parece difícil si tenemos en cuenta que el propio Lagasca $(1818,264)$ describe cómo en la zona de mayor temperatura de las hogueras a cielo abierto se forma piedra, por lo cual en los hornos barrilleros se trata de potenciar tal efecto, y de ahí su morfología: un hoyo cavado para cada fusión, de planta circular, desde 100 a $200 \mathrm{~cm}$ de diámetro, y sección ovoide, con unos 70 o $90 \mathrm{~cm}$ de profundidad. Puesto que habría de soportar el hurgoneo y la choca de la pasta de sosa durante la fusión, se buscaba para excavarlo las partes donde la tierra estaba más cohesionada y eventualmente se les daba un revoque arcilloso como refuerzo, llegando a encofrar toda la estructura si el terreno no era apto (Fig. 11). Las plantas, previamente secadas, se quemaban dentro o sobre él, dependiendo de la pericia del maestro a la hora de evitar la formación de carbones por una mala combustión, ya que éste, «sobre ocasionar una merma considerable de metal, rebaja mucho el valor de la piedra que resulta» (Lagasca, 1818, 244). A lo largo de un proceso que podía prolongarse más de 24 horas, se alternaba la quema con las operaciones para eliminar las burbujas de aire y compactar la masa, tras lo cual se cubría el horno con tierra, y se dejaba reposar

7. Se trata de «Del cultivo y aprovechamiento de la barrilla, salicor, algazul, sosa y otras plantas saladas», publicado como anexo en una reedición de la Agricultura general de Alonso de Herrera. El desinterés académico es inmemorial, a juzgar por las palabras del propio Lagasca sobre un oficio «abandonado verdaderamente al empirismo, y poco estudiado por nuestros agrónomos y naturalistas» $(1818,229)$. 


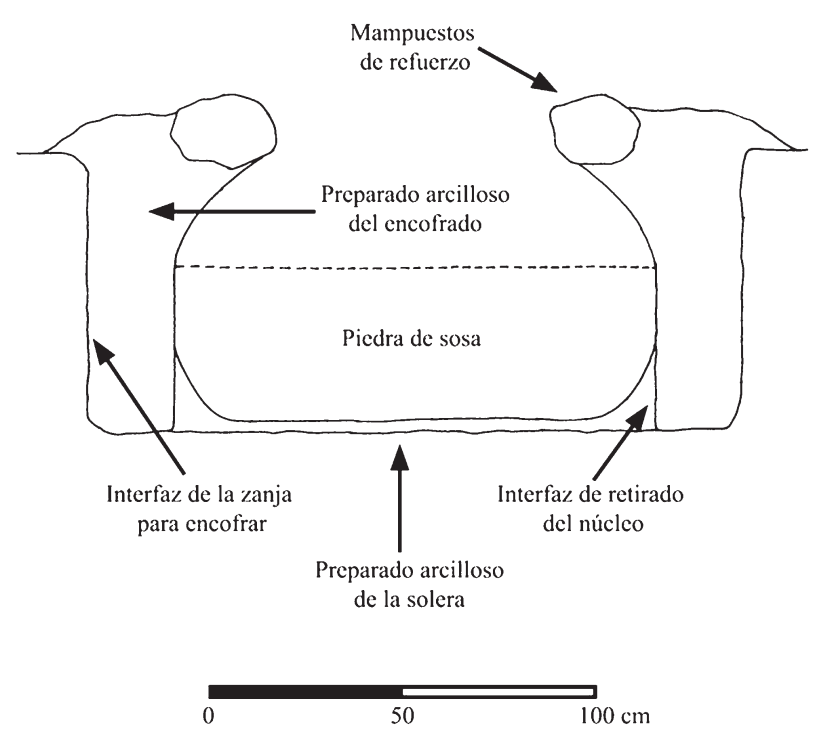

Figura 11: Sección hipotética de un horno barrillero según la descripción de Lagasca (Fernández, 1998); se trata de la variante más compleja, pero tanto forma como dimensiones se corresponden en los hornos más sencillos.

varios días esperando que solidificase correctamente, pues una fusión deficiente o una mezcla incorrecta provocaría cenizas o polvo en lugar del producto comercializable; del mismo modo, la piedra de mayor pureza se deshacía pasado cierto tiempo, pero en ambos casos se aprovechaban los restos para la siguiente quema. Por lo que toca a las mezclas, aunque Lagasca menciona también «serriche ó espato barítico, tierra de los campos y caminos, pedazos de vidrio viejo y desperdicios de las herrerías» (Lagasca, 1818, 253), lo usual era añadir en torno a un $10 \%$ de arena para «dar consistencia».

Hasta aquí contamos con un escenario económico perfectamente ajustado, el cual, además, podría incluso permitirnos una aproximación cronológica en torno a la segunda mitad del s. XVIII y no más allá de la primera del XIX, si consideramos que con el despegue comercial empieza a cultivarse y podría explicarse su producción en zonas alejadas de los focos tradicionales, más próximos a la ciudad y la costa (Lagasca, 1818, 237). En este sentido, contamos con la referencia a los Bouligny, dueños en ese momento de las tierras comprendidas entre la Foia Casans y les Índies. Además, las estructuras de combustión de Fontcalent son en principio compatibles con la descripción de Lagasca, pudiendo suponer que la mitad superior del horno se destruía al extraer el producto.

Sin embargo, existe una serie de vacíos que impiden una identificación taxativa, empezando por el elevado porcentaje de soleras que presentan, distinguibles en superficie, paquetes de tierra cenicienta y carbones ( $25 \%$ del total documentadas). Por otra parte, a la hora de extraer una piedra de sosa, de las cuales las de menor tamaño ya pesaban cerca de una tonelada (Fernández, 1998), explica Lagasca que se hacía necesario excavar una zanja más profunda que el horno a su lado, de lo cual se desprende que muy probablemente se destruía -o al menos debía de quedar afectado a un punto perfectamente reconocible en una excavación arqueológica-, lo que podría contribuir a explicar en parte la ausencia de registro arqueológico de unas estructuras que debieron de llenar vastas zonas del Camp d'Alacant, especialmente en el bien documentado litoral entre la ciudad y el Cap de l'Horta. Obviamente, tales vacíos no invalidan el total de la hipótesis, pero sí recuerdan la necesidad de enfrentar con cautela un registro que se va a demostrar todavía algo más complejo.

\section{¿HORNOS PARA LA OBTENCIÓN DE HIERRO?}

Como señalábamos en el epígrafe anterior, a las estructuras de El Oral tampoco se les ha dado una explicación enteramente satisfactoria, resultando que se menciona en su publicación la posibilidad de que estuvieran relacionadas con la deshidratación del yeso o, mejor, con algún uso metalúrgico (Abad et alii, 2001, 100) para el cual tampoco se había localizado materiales o analogías. Efectivamente, la segunda hipótesis va a seguir esta línea, abierta, en nuestro caso, por el hallazgo de escoria de reducción en el contexto de los hornos de Foia Casans II y la abundancia superficial de óxidos de hierro - principalmente oligisto $\left(\mathrm{Fe}_{2} \mathrm{O}_{3}\right)$ en todas las zonas con agrupaciones de estructuras, fruto, precisamente, de la erosión de las crestas cretácicas, ricas en este tipo de mineralizaciones, al pie de las cuales se han construido.

La cadena operativa paleosiderúrgica se divide en dos grandes etapas desde la obtención del mineral hasta el objeto elaborado. La primera convierte aquél en materia prima útil por el proceso de reducción; la segunda, identificada como post-reducción o forja, engloba los trabajos para dar forma al material metálico. Ambas responden a problemáticas específicas $\mathrm{y}$, en consecuencia, generan evidencias particulares capaces de ser rastreadas arqueológicamente hasta la reconstrucción del modelo productivo. A falta del respaldo tanto de las fuentes históricas como de un registro material contundente, no ya para la zona en torno de la sierra de Fontcalent sino para toda la región, como veremos, hemos de apoyar esta hipótesis, primero, en la analogía con algunos hornos de tecnología prehidráulica para la obtención de hierro por reducción directa, y, segundo, buscando su inserción en un modelo productivo dado, a través del repaso al conocimiento arqueológico que sobre estas prácticas se tiene para la región.

A pesar de que es uno de los elementos más comunes de la corteza terrestre, el hierro no suele darse naturalmente en su forma metálica sino en compuestos, entre los que destacan, por la rentabilización que los humanos han obtenido de ellos, los óxidos, ya que son los únicos que pueden transformarse directamente 
en hierro metálico por su reducción con carbón vegetal sin más preparaciones que los eventuales triturado, lavado y secado; esto no elimina la potencialidad de otros compuestos, pero obliga a oxidarlos previamente. De las operaciones que se verifican en el interior de un horno de reducción de hierro, la principal va a ser el paso de $\mathrm{Fe}_{2} \mathrm{O}_{3}+3 \mathrm{CO}$ a $2 \mathrm{Fe}+3 \mathrm{CO}_{2}$ a una temperatura entre 1000 y $1300{ }^{\circ} \mathrm{C}$, si bien desde los 800 se vienen dando reacciones que coadyuvan al proceso (cfr. Rovira, 1993, 57-59; Gómez, 1999, 21-22); por lo tanto, el hierro no llega a su punto de fusión con esta tecnología $\left(1536^{\circ} \mathrm{C}\right)$.

Por su parte, la tipología de estas estructuras a nivel arqueológico se complica especialmente a raíz de la desaparición del alzado de los hornos, generalmente a causa de su destrucción concluida la operación de reducción con el fin de extraer el metal del interior, de manera que no es común localizar más que las soleras, normalmente de tendencia circular u ovoide y con un diámetro variable que va de poco menos de 50 hasta $150 \mathrm{~cm}$ e incluso más ${ }^{8}$, por norma general recubierta de arcilla o algún otro material refractario. Autores como Gómez Ramos (1999, 32-33) han evidenciado la dificultad que entrañan las especulaciones sobre el alzado de los hornos, limitando su descripción a aquelo registrado positivamente; en cualquier caso, sobre la morfología, se podrían resumir las posibilidades en hornos de cubeta - por tanto, sin cubrición de obra-, de cubierta abovedada y con chimenea' ${ }^{9}$. Pero un repaso a la bibliografía disponible evidencia cierto vacío a la hora de identificar y describir tales estructuras en las intervenciones arqueológicas, en parte por la naturaleza no sólo relativamente sencilla de aquéllas sino también frecuentemente ajena a los núcleos de hábitat, resultando desproporcionada la atención que se les dedica a los subproductos respecto de los hornos que los produjeron a la hora de caracterizarlos.

Efectivamente, el otro factor fundamental en el estudio arqueológico de las tecnologías de reducción es el tipo de escorias encontradas, en función de las cuales se crea una división entre hornos «de pozo de escorias» y «de sangrado». En este segundo tipo, se dispone una piquera en la parte inferior de la solera que se rompe cuando los componentes no férricos alcanzan un estado fluido para evacuarlos fuera del horno, formando escorias globulares o de colada fácilmente identificables puesto que revelan el haber chorreado

8. Sobre el particular, a pesar de que se ha argumentado, cuestionando la posibilidad de alcanzar la temperatura de reducción con diámetros muy superiores a $50 \mathrm{~cm}$ (cfr. Burillo y Rovira, e. p., 140; Gómez, 1999, 30-32), el número de descripciones en contra de tal idea no deja lugar a dudas (cfr. Argüello, 1996, 148; Dupré y Pinçon, 2000, 96-97; Etxezarraga, 2004, 91-93).

9. Si bien estudios como los realizados en el África central, donde el paralelo etnológico ha permitido conocer de visu muchos de estos procesos y sus huellas materiales, arrojan una variabilidad enorme sobre estas formas (Dupré y Pinçon, 1997, 109-112). en estado viscoso por la superficie del suelo antes de solidificarse (Simón et alii, 1997, 221): de esta clase es el material documentado en Foia Casans II.

Si aceptamos tal identificación, su desarrollo en lo que toca a la cronología vendría a proponer a priori una horquilla entre la II Edad del Hierro y la Plena Edad Media, desapareciendo aparentemente este tipo de hornos en el momento en que ya se ha consolidado el uso de las fargas hidráulicas y en función de la verdadera brecha que crean respecto de sus predecesores en lo que concierne a optimización de recursos, por más que convivan un periodo bastante largo entre que se documentan éstas, en torno el s. X, y se generalizan, sobre el XIV (Sancho, 1996, 137-440; Urteaga, 1996, 548). En el extremo inicial de la horquilla encontramos los hornos de pozo de escorias de La Fonteta de Guardamar del Segura, efectivamente con una tecnología diferente a la que nos ocupa (Renzi y Rovira, e. p.), y una dinámica respecto del modelo productivo forzosamente marcada por el carácter singular del yacimiento fenicio. Pues bien, eliminada la Alta Edad Media, de la cual no hemos encontrado noticias para Alacant, Gómez (1999, 120 y ss., 147-148) ofrece un suficiente repaso a la situación del conocimiento para el horizonte ibérico, cuyos sitios arqueológicos y problemáticas a la postre se solapan en buena parte con los de la Antigüedad clásica.

En definitiva, dicho autor viene a evidenciar que faltan los estudios en profundidad y las noticias, prácticamente todas provenientes de contextos poblacionales, son muy confusas, refiriéndose a «escorias» sin mayor descripción y sin una vinculación clara con las estructuras donde se han generado, muchas veces no localizadas; de hecho, también se abordó la cuestión en la III Reunió sobre economia al món ibèric (Mata et alii, 2000, 209-227, 249-309), resultando que, las más de las veces, las evidencias de los asentamientos se circunscriben a los procesos de post-reducción. Y es lógico que fuera de este modo, mientras que para las reducciones se priorizara la proximidad a unos recursos pesados o voluminosos, buena parte de los cuales, además, se desecharía tras verificar la operación. En este sentido se expresaba, refiriéndose a la metalurgia de la plata en el contexto de El Oral, el geólogo César Doménech en el addendum de un artículo de Abad y Sala Sellés (1993, 203), y, en efecto, es la situación que se ha demostrado en regiones como el País Vasco para la paleosiderurgía romana y medieval (Etxezarraga, 2004; Franco Pérez, 2007, 2008a y b).

Desde esta óptica resulta paradigmático el caso del Rincón de la Mina de Tuéjar, a la postre la única cita fuera de poblado de las evidencias recopiladas por Gómez. Se trata de una explotación de hierro en galería, en cuya boca se localizaron «cantidades ingentes» de escoria, tanto como en el cercano Campo de las Herrerías, para el cual el autor de la noticia supone la existencia de «varias fundiciones, bien simultáneas o trasladadas sucesivamente de sitio, conforme se iban agotando los bosques circundantes» (Palomares, 

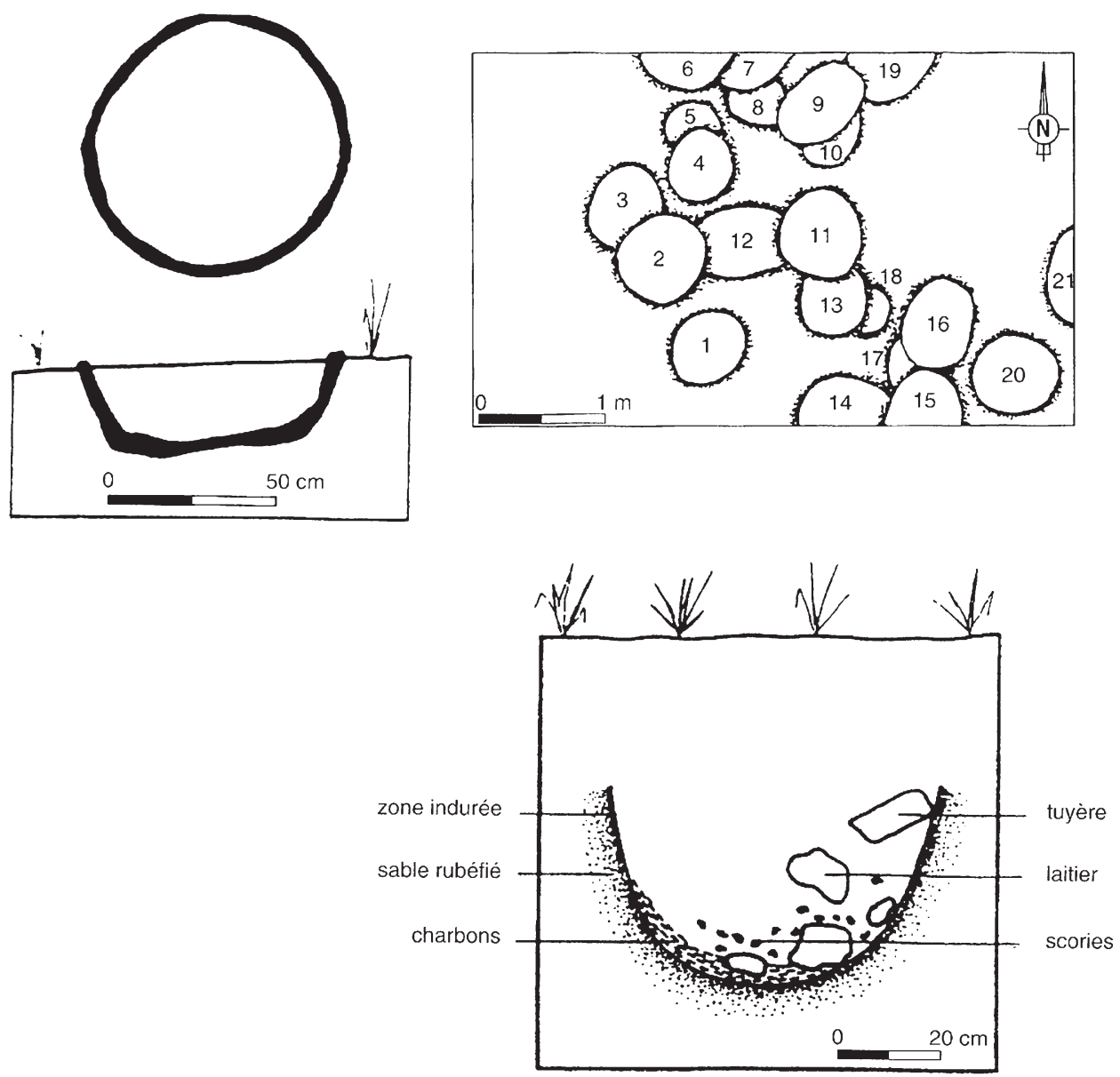

Figura 12: Planta y sección de los restos de hornos siderúrgicos en Loukoko, fechados hacia 1910 BP (arriba), y Kankouara I, hacia 870 BP (derecha, la sección corresponde al horno 20), ambos en el África central (Dupré y Pinçon, 1997, 58).

1966, 242-246). Por desgracia, no tenemos noticia de que se haya vuelto a trabajar sobre la zona desde 1966, y carecemos de descripciones de las estructuras que generaron dichas escorias, si es que se identificaron.

En este marco, Fontcalent y los afloramientos que la circundan cumplen el requisito de lugar con abundancia de mineralizaciones de hierro en superficie, y no sólo es uno de los pocos de la comarca -junto con la zona del Cabeçó d'Or y el arco montañoso que la cierra hasta el Savinar-, sino que además es un hito referencial y un cruce de caminos, con disponibilidad de agua y combustible para la cronología que ha de suponérseles a los hornos. Por tanto, es asimismo posible un escenario inserto en una economía preindustrial y más sujeta al territorio, en la cual la demanda de hierro se satisficiera antes con una explotación ligera y más o menos puntual que por medio de la importación desde hipotéticos centros especializados alejados muchos kilómetros, con el riesgo que supone la posibilidad de comprometer el abastecimiento de un producto estratégico para el éxito del grupo humano. En tal tipo de explotación las reducciones se realizan desplazándose cuando es necesario hasta los afloramientos de mineral y recogiendo el suficiente en superficie, levantando un horno de obra relativamente ligera y abandonándolo tras la operación, resultando de la recurrencia de este proceso la aparición de agrupaciones de hornos diseminadas en una amplia zona (Fig. 12): se trata de un modelo productivo documentado en todos sus puntos en sociedades como las del África central, estudiadas por Dupré y Pinçon ${ }^{10}$, y occidental, por Martinelli ${ }^{11}$.

Encontramos, por tanto, que esta segunda hipótesis puede respaldarse con un modelo productivo marco viable para una región en la que se carece de estudios al respecto. Pero no está exenta de problemas, y graves, pues precisamente la falta de estudios nos deja como referente bien documentado más cercano los hornos vizcaínos, cuyas soleras son más duras y resuelven sus alzados habitualmente en piedra, sin entrar a contar la cantidad incomparablemente mayor de escorias que los rodean. $\mathrm{Y}$ en estas circunstancias es

10. Para la periodicidad de las reducciones y el abastecimiento de mineral, especialmente 1997, 140-146.

11. Para el abastecimiento de mineral, 1982, 39 y ss.; para las diferentes articulaciones sociales que sustentan estos modelos productivos, 2000, 126-130. 


\begin{tabular}{|c|r|r|r|r|}
\hline Elemento & Horno A2 & Testigo A & Horno D1 & Testigo D \\
\hline $\mathrm{Ba}$ & 38,5 & 36,9 & 63,7 & 57,8 \\
\hline $\mathrm{Ca}$ & 73306,0 & 78573,3 & 59179,1 & 71938,2 \\
\hline $\mathrm{K}$ & 310,2 & 244,3 & 574,0 & 647,6 \\
\hline $\mathrm{Fe}$ & 4838,5 & 5500,3 & 20348,3 & 13441,2 \\
\hline $\mathrm{Li}$ & 16,6 & 21,9 & 45,7 & 55,8 \\
\hline $\mathrm{Mg}$ & 5757,8 & 5456,9 & 5434,1 & 5588,5 \\
\hline $\mathrm{Mn}$ & 37,7 & 47,2 & 138,6 & 150,9 \\
\hline $\mathrm{Na}$ & Saturado & 272,3 & 315,4 & 304,0 \\
\hline $\mathrm{P}$ & 24,0 & 24,7 & 66,6 & 81,1 \\
\hline $\mathrm{Si}$ & 137,2 & 325,3 & 383,3 & 208,2 \\
\hline $\mathrm{Sr}$ & 356,3 & 344,5 & 207,3 & 214,4 \\
\hline $\mathrm{Ti}$ & 376,8 & 389,7 & 375,0 & 376,5 \\
\hline $\mathrm{V}$ & 19,2 & 23,3 & 45,1 & 50,6 \\
\hline
\end{tabular}

Figura 13: Tabla comparativa de los $\mathrm{mg} / \mathrm{kg}$ según elemento y muestra; se presentan únicamente aquellos cuyos valores sobrepasan los $50 \mathrm{mg} / \mathrm{Kg}$ en al menos una de las muestras.

imposible afirmar que nos encontramos ante una solución constructiva adaptada a las condiciones regionales, marcadas además por la fuerte afección de los procesos postdeposicionales de pérdida de un registro relativamente débil de por sí, tanto como descartar tal posibilidad.

\section{ALGUNAS (IN)CONCLUSIONES}

Como veníamos diciendo, el registro material proporcionado por las estructuras de combustión de Fontcalent va a mostrarse todavía algo más complejo, y si con la metodología prevista se ha alcanzado un punto en el cual los ajustes hacia una u otra hipótesis empiezan a reclamar evidencias determinantes, tampoco la posibilidad, surgida poco antes de cerrar el presente trabajo, de realizar unos primeros análisis químicos de tierras (ICP-MS) ${ }^{12}$ ha acabado por esclarecer el uso de los hornos. Por otra parte, hay que indicar que también en este caso carecíamos de pruebas análogas con las que comparar los resultados obtenidos, ya porque la obviedad de los escoriales de reducción de hierro hace innecesarios los análisis químicos, ya directamente porque no se han documentado hornos barrilleros arqueológicamente. Sin embargo, tales procesos productivos generan en mayor o menor medida una alteración

12. He de agradecer esta posibilidad a $\mathrm{M}^{\mathrm{a}}$ Victoria Rigo y al equipo del laboratorio del Centro de Salud Pública de Alacant, donde se realizaron las pruebas. Consistieron en la digestión ácida (ácido nítrico 65\% y ácido clorhídrico $37 \%$ ) asistida por microondas (sistema cerrado a presión a una temperatura de $190{ }^{\circ} \mathrm{C}$ ) de 0,2 gr de muestra en 3 $\mathrm{ml}$ de $\mathrm{HNO}_{3}$ c y $1 \mathrm{ml}$ de $\mathrm{HCl}$ c, sometiendo al resultado a un análisis mediante espectrometría de masas con plasma de acoplamiento inductivo (ICP-MS) en modo semicuantitativo (concentraciones más o menos $20 \%$ del valor obtenido); los patrones internos utilizados fueron rutenio, teluro y renio. en la composición química del lugar en el que se verifican respecto del resto del terreno circundante, y esto es lo que se trataba de medir.

Con este fin, se recogieron cuatro muestras de tierra correspondientes a los paquetes cenicientos del interior de las soleras A2 y D1, y dos más unos 10$20 \mathrm{~m}$ aguas arriba de sendas estructuras, a modo de testigos. Una desproporción acusada en los niveles de sodio $(\mathrm{Na})$ de las muestras de las soleras respecto de las del exterior debería señalar, en condiciones ideales de análisis, hacia la primera hipótesis, mientras que su equilibro complicaría esta caracterización; pero con lo que no se contaba era con unos resultados diametralmente opuestos para cada estructura, tal y como ha revelado el análisis (Fig. 13). Así, si efectivamente la saturación de sodio en A2 pudiera explicarse posiblemente con un uso vinculado a la producción de piedra de sosa, el hecho de registrar no sólo unos porcentajes muy próximos para dicho elemento entre D1 y su testigo, sino especialmente que en este segundo caso el desequilibrio se recoja en un porcentaje próximo al $150 \%$ de hierro $(\mathrm{Fe})$ en el interior del horno respecto de su testigo, nos devuelve prácticamente a las condiciones anteriores al análisis. Por su parte, la acusada caída del calcio $(\mathrm{Ca})$ en D1 o las tendencias opuestas del silicio ( $\mathrm{Si}$ ) entre éste y A2 redundan en la disparidad de resultados, pero -como el nivel de hierro-, sin el respaldo sólido de estudios similares en estructuras previamente caracterizadas, tampoco ayudan a identificar por sí solas el proceso productivo para el que fueron construidos estos hornos; identificación ahora dificultada al poder cuestionarse incluso el apriorismo por el cual unas estructuras morfológicamente iguales localizadas en una misma zona debían ser el fruto de una única actividad.

Con únicamente dos análisis para más de 15 soleras localizadas, si un resultado uniforme sólo quizá hubiera ayudado a respaldar una interpretación funcional, con lo expuesto lo que viene a respaldarse sin 
duda es la necesidad de profundizar en el estudio de estos restos. Opinamos que se ha generado una base reflexiva suficiente como para agotar las posibilidades de una intervención arqueológica ligera focalizada sobre la zona inmediata, como la llevada a cabo, y que la necesidad de minimizar las suposiciones obliga, en primer término, a conocer mejor estas estructuras mediante una intervención más completa. Pues si algo ha quedado demostrado es el interés que encierran los hornos dispersos en el entorno de la sierra de Fontcalent, sea cual fuere su explicación, como evidencia de una actividad económica importante para los grupos humanos que habitaron el Camp d'Alacant.

\author{
Jordi A. López Lillo \\ Área de Arqueología \\ Dpto. Prehistoria, Arqueología, $\mathrm{H}^{\mathrm{a}}$ Antigua, \\ Filología Griega y Filología Latina \\ Facultad de Filosofía y Letras \\ Universidad de Alicante \\ 03080 Alicante \\ jordi.lopez@ua.es
}

\section{BIBLIOGRAFÍA}

AA.VV., 2008a: Actuaciones arqueológicas en la Provincia de Alicante, 2006, Alicante.

AA.VV., 2008b: Actuaciones arqueológicas en la Provincia de Alicante, 2007, Alicante.

ABAD CASAL, L. y SALA SELLÉS, F., 1993: «Reflexiones sobre la metalurgia protohistórica: el poblado ibérico de El Oral (San Fulgencio, Alicante)», en R. ARANA CASTILLO (coord.), Metalurgia en la Península Ibérica durante el primer milenio a. C.: estado actual de la investigación, Murcia, 189-204.

ABAD CASAL, L. y SALA SELLÉS, F. (eds.), 2001: Poblamiento ibérico en el Bajo Segura. El Oral (II) y La Escuera, Madrid.

ARGÜELLO MENÉNDEZ, J. J., 1996: «Minería y metalurgia en Muñás y Carceu (Valdés, Asturies)», en Actas de las I Jornadas sobre minería y tecnología en la Edad Media peninsular, León, 134-161.

AZUAR RUIZ, R. e HINOJOSA MONTALVO, J. (Coords.), 1990: Historia de la ciudad de Alicante, tomo II: Edad Media, Alicante.

BENDICHO, V. 1650: Chronica de la muy ilustre, noble y leal ciudad de Alicante, Edición a cargo de $\mathrm{M}^{\mathrm{a}}$ Luisa Cabanes Catalá, 1991, Alicante.

BURILLO MOZOTA, F. y ROVIRA LLORENS, S. (en prensa): «Experimentos de fundición de minerales de hierro en la ciudad-estado celtibérica de Segeda (Mara, Zaragoza)», en Avances en Arqueometría: 2005, Actas del VI Congreso Ibérico de Arqueometría, 137-147.

DUPRÉ, M.-CL. y PINÇON, B., 1997: Métallurgie et politique en Afrique centrale. Deux mille ans de vestiges sur les plateaux batéké (Gabon, Congo, Zaïre), París.

DUPRÉ, M.-CL. y PINÇON, B., 2000: «La métallurgie, science humaine: 2500 ans d'artisanat en Afrique centrale atlantique», en P. PÉTREQUIN, P. FLUZIN, J. THIRIOT y P. BENOIT (eds.), Arts $d u$ feu et productions artisanales, XXe Rencontres Internationales d'Archéologie et d'Histoire d'Antibes, Antibes, 87-106.

ETXEZARRAGA, I., 2004: «Paleometalurgia del hierro en el País Vasco Cantábrico: las haizeolak. Un estado de la cuestión», Munibe, 56, 87-104.

FERNÁNDEZ PÉREZ, J. y GONZÁLEZ TASCÓN, I., 1990: «Las plantas barrilleras. La obtención de la sosa y la potasa», en J. HERNÁNDEZ e I. GONZÁLEZ (eds.), La agricultura viajera. Cultivos y manufacturas de plantas industriales y alimentarias en España y en la América Virreinal, Madrid, 213-237.

FERNÁNDEZ PÉREZ, J., 1998: «La elaboración de la sosa en España: de la barrilla a la fábrica Solvay de Torrelavega», Antilia: Revista española de historia de las ciencias de la naturaleza y de la tecnología, IV, art. 1.

FRANCO PÉREZ, F. J., 2007: «Nuevas propuestas de prospección arqueológica en la región cantábrica: el caso de las ferrerías de monte de Vizcaya», Territorio, Sociedad y Poder: Revista de Estudios medievales, 2, 37-52.

FRANCO PÉREZ, F. J., 2008a: «Carta arqueológica de las ferrerías de monte de Bizkaia, últimas investigaciones», VII Congreso Internacional sobre Patrimonio Geológico y Minero: libro de actas, Puertollano, 417-430.

FRANCO PÉREZ, F. J., 2008b: «Ferrerías de monte en los macizos de Ganekogorta, Beraskola y Oiz (Alonsotegi, Bilbao)», Arkeoikuska 2007, 309-313.

GARCÍA GARIJO, E., 1987: «La Alcoraya: un espacio histórico agrícola y vial», Sharq Al-Andalus: Estudios mudéjares y moriscos, 4, 153-158.

GIL OLCINA, A., 1975: «Explotación y cultivo de las plantas barrilleras en España», Estudios geográficos, 36, núm. 138-139, 453-478.

GÓMEZ RAMOS, P., 1999: Obtención de metales en la Prehistoria de la Península Ibérica, Oxford.

GUTIÉRREZ LLORET, S., 1986: «Cerámicas comunes altomedievales: contribución al estudio del tránsito de la Antigüedad al mundo paleoislámico en las comarcas meridionales del País Valenciano», Lucentum, V, 147-167.

HERNÁNDEZ PÉREZ, M. S., 1989: «La Prehistoria», en F. MORENO (dir.), Historia de Alicante, tomo I, Alicante, 41-60.

INSTITUTO GEOLÓGICO Y MINERO DE ESPAÑA, 1978: Mapa Geológico de España e. 1:50.000, Hoja 871 28-34, correspondiente a Elda, y su Memoria adjunta, Madrid.

LAGASCA Y SEGURA, M., 1818: «Del cultivo y aprovechamiento de la barrilla, salicor, algazul, sosa y otras plantas saladas», en D. ALONSO DE HERRERA, Agricultura general, tomo I, Edición facsímil virtual del Real Jardín Botánico-CSIC, 228-312.

LLOBREGAT CONESA, E. A., 1970: «Materiales hispanovisigodos del Museo Arqueológico Provincial de Alicante», en Papeles del Laboratorio de Arqueología de Valencia, 10, 189-204.

LLOBREGAT CONESA, E. A., 1977: La primitiva cristiandat valenciana. Segles IV al VIII, Valencia.

LLOBREGAT CONESA, E. A., 1983: «Relectura del Ravennate: dos calzadas, una mansión inexistente y otros 
datos de la geografía antigua del País Valenciano», $L u$ centum, II, 225-242.

LÓPEZ SEGUÍ, E., 1996: Arqueología en Agost (Alicante), Alicante.

MADOZ, P., 1845-1850: Diccionario geográfico-estadistico-histórico de España y sus posesiones de Ultramar, tomo I, Edición facsímil virtual de la Universidad de Alicante.

MARTINELLI, B., 1982: Métallurgistes bassar. Techniques et formation sociale, Benin.

MARTINELLI, B., 2000: «Le choix de la combustion lente. Mutation technique et mutation sociale au Yatenga, Burkina Faso», en P. PÉTREQUIN, P. FLUZIN, J. THIRIOT y P. BENOIT (eds.), Arts du feu et productions artisanales, XXe Rencontres Internationales d'Archéologie et d'Histoire d'Antibes, Antibes, 123-142.

MATA PARREÑO, C. y PÉREZ JORDÁ, G. (Eds.), 2000: Íbers. Agricultors, artesans i comerciants: III Reunió sobre Economia en el Món Ibèric, Saguntum, extra 3, Valencia.

MILLARES CANTERO, A., 1982: «Arrecife, el puerto de la barrilla (En torno a los orígenes de una ciudad burguesa canaria entre el Antiguo y el Nuevo Régimen)», Boletín Millares Carlo, 5, 67-160.

MORATALLA JÁVEGA, J., 2005: «El territorio meriodional de la Contestania», en L. ABAD, F. SALA e I. GRAU (eds.), La Contestania ibérica, treinta años después, Alicante, 91-117.

PALENCIA PÉREZ, R., 2007: Francisco Bouligny, un alicantino en la colonización de Luisiana, Alicante.

PALOMARES, E., 1966: «Hallazgos arqueológicos de Sinarcas y su comarca», Archivo de Prehistoria Levantina, XI, 231-247.

POVEDA NAVARRO, A. M., 1991: «Transformación y romanización del hábitat ibérico contestano de las cuencas alta y media del Vinalopó (provincia de Alicante): del final de la República al Alto Imperio», Alebus: Cuadernos de estudios históricos del valle de Elda, 1, 65-78.

RENZI, M. y ROVIRA LLORENS, S. (en prensa): «Escorias metalúrgicas del yacimiento fenicio de La Fonteta (Alicante). Estudio preliminar», en Avances en Arqueometría: 2005, Actas del VI Congreso Ibérico de Arqueometría, 163-171.

REYNOLDS, P., 1985: «Cerámica tardorromana modelada a mano de carácter local, regional y de importación en la Provincia de Alicante», Lucentum, IV, 245-267.
RIBES, V., 1994-1995: «Bouligny, la vigília de la raó», Quaderns de Migjorn: Revista d'estudis comarcals del sud del País Valencià, 2, 87-92.

ROVIRA LLORENS, S., 1993: «La metalurgia de la Edad del Hierro en la península ibérica: una síntesis introductoria», en R. ARANA CASTILLO (coord.), Metalurgia en la Península Ibérica durante el primer milenio a. C.: estado actual de la investigación, Murcia, 45-70.

SALA CAJA, L., 2003: «La competencia terminológica: causas lingüísticas en el auge del término sosa y el declive de barrilla en los siglos XVIII y XIX», Asclepio: Revista de historia de la medicina y de la ciencia, LV: 2, 67-92.

SANCHO I PLANAS, M., 1996: «Aportaciones de la arqueología para el estudio de la producción de hierro en la Cataluña medieval: estado de la investigación y nuevas perspectivas», en Actas de las I Jornadas sobre minería y tecnología en la Edad Media peninsular, León, 436-450.

SIMÓN ARIAS, J., MARSAL ASTORT, M., PÉREZ SUÑÉ, J. y GÓMEZ SÁNCHEZ, J., 1997: «Caracterización de las escorias de hierro y su interpretación en el contexto histórico arqueológico», Caesaraugusta, 73, II Congreso Nacional de Arqueometría, 217-227.

SOLER DÍAZ, J. A., 2002: Cuevas de inhumación múltiple en la Comunidad Valenciana, vol. I, Madrid-Alicante.

UROZ SÁEZ, J., 1990: «L'Alcoraya (Alacant)», en Excavacions arqueológiques de salvament a la Comunitat Valenciana, 1984-1988, tomo II: Intervencions rurals, Valencia, 13-14.

URTEAGA ARTIGAS, M. M., 1996: «Siderurgia medieval en Gipuzkoa. Haizeolas, ferrerías masuqueras y ferrerías mazonas», en Actas de las I Jornadas sobre minería y tecnología en la Edad Media peninsular, León, 543-554.

VELASCO HERNÁNDEZ, F., 2004: «La sosa-barrilla: una seña de identidad del Campo de Cartagena en los siglos XVI y XIX», en Revista Murciana de Antropología, 10, 145-158.

VIRAVENS Y PASTOR, R., 1876: Crónica de la muy ilustre y siempre fiel ciudad de Alicante, Edición facsímil de 1986, Alicante.

XIMÉNEZ DE EMBÚN SÁNCHEZ, M. T., 2007: El Camp d'Alacant en la Alta Edad Media. Una nueva mirada sobre el poblamiento Tardio en tierras alicantinas. Tesina, Universidad de Alicante. 